

\title{
Elliptic nets and elliptic curves
}

\author{
Katherine Stange
}

An elliptic divisibility sequence is an integer recurrence sequence associated to an elliptic curve over the rationals together with a rational point on that curve. In this paper we present a higher-dimensional analogue over arbitrary base fields. Suppose $E$ is an elliptic curve over a field $K$, and $P_{1}, \ldots, P_{n}$ are points on $E$ defined over $K$. To this information we associate an $n$-dimensional array of values in $K$ satisfying a nonlinear recurrence relation. Arrays satisfying this relation are called elliptic nets. We demonstrate an explicit bijection between the set of elliptic nets and the set of elliptic curves with specified points. We also obtain Laurentness/integrality results for elliptic nets.

Introduction

1. Elliptic nets 200

2. Laurentness and integrality 201

3. Net polynomials over $\mathbb{C} \quad 210$

4. Net polynomials over arbitrary fields 215

5. Elliptic nets from elliptic curves 219

6. Elliptic curves from elliptic nets 221

7. The curve-net theorem 225

Acknowledgements 228

References $\quad 228$

\section{Introduction}

An elliptic divisibility sequence is an integer sequence $W_{n}$ satisfying

$$
W_{n+m} W_{n-m}=W_{n+1} W_{n-1} W_{m}^{2}-W_{m+1} W_{m-1} W_{n}^{2} .
$$

This definition was introduced by Morgan Ward [1948]. Let $\Psi_{n}(x, y)$ be the $n$-th division polynomial associated to an elliptic curve (the $n$-th division polynomial vanishes at the $n$ torsion points). Ward showed that division polynomials satisfy

This work was supported by NSERC Awards PGS D2 331379 and PDF 373333.

MSC2000: primary 11G05, 11G07, 11B37; secondary 11B39, 14H52.

Keywords: elliptic net, elliptic curve, Laurentness, elliptic divisibility sequence, recurrence sequence. 
the recurrence (1) and furthermore that all elliptic divisibility sequences have the form

$$
W_{n}=\lambda^{n^{2}-1} \Psi_{n}(x, y)
$$

for some constant $\lambda$, elliptic curve (or singular cubic) and point $P=(x, y)$ on the curve. This rich structure has led to number-theoretic results [Ayad 1993; Everest et al. 2006; Ingram 2009; Silverman 2004; 2005; Swart 2003] and to applications to Hilbert's Tenth Problem [Cornelissen and Zahidi 2007; Eisenträger and Everest 2009; Poonen 2003], to integrable systems [Hone 2005], and to cryptography [Chudnovsky and Chudnovsky 1986; Shipsey 2001; Stange 2007]. For a bibliography, see [Everest et al. 2003, Chapter 10].

There have been several attempts to generalize this theory. Translated elliptic divisibility sequences were studied in [Swart 2003; van der Poorten 2005; van der Poorten and Swart 2006]. Mazur and Tate [1991] generalize division polynomials to arbitrary endomorphisms in the p-adic setting, and Streng [2008] uses their definition to generalize to the endomorphism ring of an elliptic curve with complex multiplication. Elliptic divisibility sequences are closely related to the denominators of the multiples $[n] P$ of a fixed point $P$; questions have been asked about the collection of denominators of the linear combinations $[n] P+[m] Q$ by Everest, Miller and Stephens [2004]. The hope of defining higher-rank elliptic divisibility sequences via a recurrence relation was discussed in correspondence by Elkies, Propp and Somos [Propp 2001].

The primary purpose of this paper is to generalize from integer sequences to multidimensional arrays with values in any field, which we call elliptic nets. A substantial part of the difficulty lies in finding the correct recurrence and defining a generalized division polynomial.

We define an elliptic net to be a function $W: A \rightarrow R$ from a finite-rank free abelian group $A$ to an integral domain $R$ satisfying the properties that $W(0)=0$ and that

$$
\begin{aligned}
& W(p+q+s) W(p-q) W(r+s) W(r) \\
& +W(q+r+s) W(q-r) W(p+s) W(p) \\
& +W(r+p+s) W(r-p) W(q+s) W(q)=0
\end{aligned}
$$

for all $p, q, r, s \in A$. If $A=R=\mathbb{Z}$, this is an equivalent definition of an elliptic divisibility sequence (this is not immediately obvious, but it is a consequence of results in this paper). By the rank of an elliptic net we shall mean the rank of $A$ (this bears no relation to the rank of apparition defined in [Ward 1948] for elliptic divisibility sequences). Section 1 covers the basic definitions and gives examples.

Our primary interest is the relationship between elliptic curves and elliptic nets. 
Main Theorem (introductory version). For each field $K$ and integer $n$, there is an explicit bijection of sets

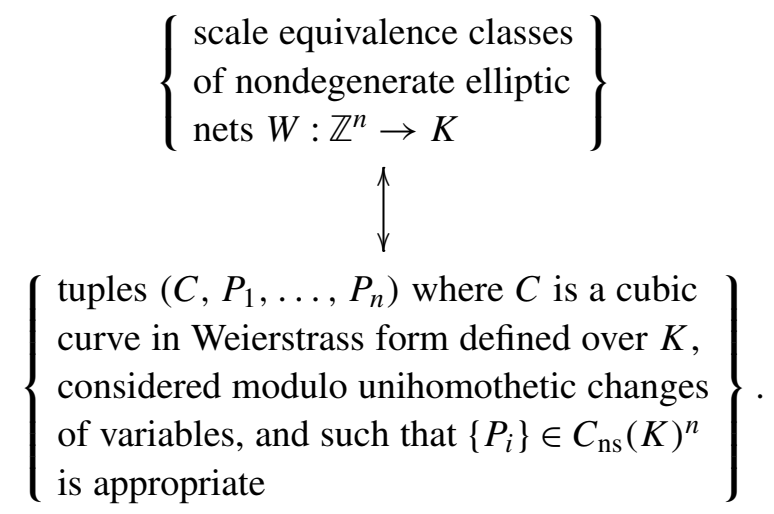

For a description of the relevant terminology, see Section 5 (appropriate), page 221 (scale equivalent, nondegenerate) and page 222 (unihomothetic). See Theorem 7.4 for a more detailed statement. The isomorphism itself is described explicitly in Definition 5.1 (depending on Theorem 4.6) and Theorem 6.7. For ranks 1 and 2, explicit formulae can be found in Propositions 3.8, 6.3 and 6.4. For an example, see (4).

The other main aspect of elliptic nets studied in this paper is Laurentness. These results are needed for the proof of the main theorem, but are of independent interest. One property of elliptic divisibility sequences of particular interest is that they are integer sequences: if the sequence begins $1, a, b, a c, \ldots(a, b, c \in \mathbb{Z})$, then it will consist entirely of integers [Ward 1948]. This result has been studied in the more general framework of the Laurent phenomenon of [Fomin and Zelevinsky 2002].

Laurentness results are found in Section 2, which is devoted to the inductive structure of elliptic nets: how some terms are determined by others via the recurrence relation. We define a universal ring $\mathcal{W}_{A}$ for elliptic nets on $A$, such that elliptic nets $W: A \rightarrow R$ are in bijection with homomorphisms $\mathcal{W}_{A} \rightarrow R$. We obtain results on the structure of this ring, and in turn, these imply integrality results. See Theorem 2.2 for the case $n=1$, Theorem 2.5 for $n=2$, and Theorem 2.8 for $n \geq 3$. The proofs in this section are elementary but somewhat tedious. The author has not been successful in replacing them with methods similar to those of [Fomin and Zelevinsky 2002], although the possibility remains.

The next two sections define the higher-rank generalization of division polynomials called net polynomials: rational functions on the $n$-fold product $E^{n}$ of an elliptic curve $E$, which vanish on tuples $\left(P_{1}, \ldots, P_{n}\right)$ satisfying a linear relation $\left[v_{1}\right] P_{1}+\cdots+\left[v_{n}\right] P_{n}=0$ for fixed coefficients $v_{i}$. In Section 3, we work with the complex uniformization of an elliptic curve defined over $\mathbb{C}$. In Section 4 we 
generalize the definition to arbitrary fields by analysing the arithmetic properties of net polynomials. The main result here is Theorem 4.4.

The last three sections describe the bijection in the main theorem. Section 5 makes explicit the production of an elliptic net from any cubic Weierstrass curve using the net polynomials. Section 6 determines exactly those cubic curves which produce a given elliptic net. Finally, Section 7 puts together the results of the previous sections to prove the main theorem, stated in its full form as Theorem 7.4.

Computer software. The explicit isomorphism described in this paper has been implemented for Pari/GP and SAGE in ranks 1 and 2; see [Stange 2010].

\section{Elliptic nets}

Definition 1.1. Let $A$ be a free finitely-generated abelian group and $R$ an integral domain. An elliptic net is any map $W: A \rightarrow R$ with

$$
W(0)=0,
$$

and such that, for all $p, q, r, s \in A$,

$$
\begin{aligned}
& W(p+q+s) W(p-q) W(r+s) W(r) \\
& \quad+W(q+r+s) W(q-r) W(p+s) W(p) \\
& \quad+W(r+p+s) W(r-p) W(q+s) W(q)=0 .
\end{aligned}
$$

Functions $W: A \rightarrow R$ which satisfy (3) but not (2) can only appear in characteristic 3 (to see this, take $p=q=r=s=0$ in (3)). Any constant function in characteristic 3 is an example. By definition, these are not elliptic nets.

We refer to the rank of $A$ as the rank of the elliptic net. Suppose that $B \subset A$ is a subgroup of $A$. Then the restriction to $B$ of an elliptic net $W: A \rightarrow R$ is also an elliptic net. We refer to this elliptic net as the subnet associated to $B$ and write $\left.W\right|_{B}: B \rightarrow R$.

Example 1.2. Let $R$ be an integral domain. The following are elliptic nets.

- The zero net $W: \mathbb{Z}^{n} \rightarrow R$ defined by $W(\boldsymbol{v})=0$ for all $\boldsymbol{v}$.

- The identity map $W_{i d}: \mathbb{Z} \rightarrow \mathbb{Z}$ given by $W(v)=v$.

- Let $W^{\prime}: \mathbb{Z} \rightarrow R$ be an elliptic net. Then for each $1 \leq i \leq n$, we may define $W_{i}: \mathbb{Z}^{n} \rightarrow R$ by $W_{i}\left(v_{1}, \ldots, v_{n}\right)=W^{\prime}\left(v_{i}\right)$, and this will also be an elliptic net.

- More generally, if $W: A \rightarrow R$ is an elliptic net and $f: B \rightarrow A$ is a homomorphism of finitely generated free abelian groups, then $W \circ f: B \rightarrow R$ is also an elliptic net.

- If $W: A \rightarrow R$ is an elliptic net and $g: R \rightarrow S$ is a homomorphism of integral domains, then $g \circ W: A \rightarrow S$ is also an elliptic net. 
- $W_{\text {Leg }}: \mathbb{Z} \rightarrow \mathbb{Z}$ given by $W(v)=\left(\frac{v}{3}\right)$, the Legendre symbol of $v$ over 3 . This can be verified by a finite examination of cases; observe that at least one of $p$, $q, r, p-q, q-r$, and $r-p$ is divisible by 3. See also [Ward 1948, p. 31].

- $W_{\mathrm{Fib}}: \mathbb{Z} \rightarrow \mathbb{Z}$ given by

$$
W(v)=\left\{\begin{array}{cl}
F_{2 v} & \text { if } v>0 \\
-F_{2 v} & \text { if } v<0 \\
0 & \text { if } v=0
\end{array}\right.
$$

where $F_{2 v}$ is the $2 v$-th Fibonacci number. One checks this example using the closed form for terms of the Fibonacci sequence. See also [Ward 1948, p. 31].

- Here is a portion of an elliptic net of rank 2, displayed as an array:

\begin{tabular}{|c|c|c|c|c|c|c|c|c|}
\hline 3269 & -2869 & 4335 & 5959 & 12016 & -55287 & 23921 & 1587077 & -7159461 \\
\hline-127 & -299 & 94 & 479 & 919 & -2591 & 13751 & 68428 & 424345 \\
\hline-44 & -27 & -31 & 53 & -33 & -350 & 493 & 6627 & 48191 \\
\hline-1 & -7 & -5 & 8 & -19 & -41 & -151 & 989 & -1466 \\
\hline 3 & -2 & 1 & 3 & -1 & -13 & -36 & 181 & -1535 \\
\hline 1 & -1 & 1 & 1 & 2 & -5 & 7 & 89 & -149 \\
\hline-1 & -1 & 0 & 1 & 1 & -3 & 11 & 38 & 249 \\
\hline-2 & -1 & -1 & 1 & -1 & -4 & 1 & 47 & 185 \\
\hline 1 & -3 & -1 & 2 & -3 & -5 & -17 & 63 & -184 \\
\hline
\end{tabular}

This example arises from the curve $y^{2}+y=x^{3}+x^{2}-2 x$ over $\mathbb{Q}$ and the two points $P=(0,0), Q=(1,0)$; see Example 5.3. The origin is at the term with value 0 . Each axis forms an elliptic divisibility sequence, e.g., $0,1,1$, $-3,11,38,249, \ldots$.

\section{Laurentness and integrality}

In this section we ask which terms of an elliptic net determine the others via the recurrence relation. In the case of $n=1$, Ward [1948] showed that the terms $W(1), \ldots, W(4)$ sufficed to determine the rest of the net (unless too many of these terms were zero). Our method also demonstrates Laurentness and integrality results. The main theorems of this section are used in Section 6.

Laurentness. Let $I$ be a group, in additive notation, called the indexing group, whose elements are called indices. To each $i \in I$, we associate the symbol $T_{i}$. In what follows, the indexing group will be $I \cong \mathbb{Z}^{n}$ for some $n$.

Consider the ideal $\mathcal{M}$ in the ring $\mathbb{Z}\left[T_{i}\right]_{i \in I}$ generated by $T_{0}$ and all polynomials encoding property (3), i.e., those of the form

$$
T_{p+q+s} T_{p-q} T_{r+s} T_{r}+T_{q+r+s} T_{q-r} T_{p+s} T_{p}+T_{r+p+s} T_{r-p} T_{q+s} T_{q}
$$


as $p, q, r, s$ range over $I$. Polynomials of the form (5) will be called recurrence relations. Consider the ring $\mathcal{W}_{I}$ obtained from $\mathbb{Z}\left[T_{i}\right]_{i \in I} / \mathcal{M}$ as a quotient by its own nilradical. For each integral domain $R$, there is a bijection between elliptic nets $W: I \rightarrow R$ and homomorphisms $\mathcal{W}_{I} \rightarrow R$ (defined by taking $T_{i} \mapsto W(i)$ ).

Taking $p=q=i, r=s=0$ shows that $T_{i}^{3}\left(T_{i}+T_{-i}\right) \in \mathcal{M}$ for each $i \in I$. In particular, $T_{-i}^{3}\left(T_{i}+T_{-i}\right) \in \mathcal{M}$ also. Therefore, any prime ideal containing $\mathcal{M}$ contains $T_{i}+T_{-i}$; for if it did not, then it must contain $T_{i}$ and $T_{-i}$, a contradiction. Therefore $T_{-i}=-T_{i}$ in $\mathscr{W}_{I}$. This implies the following.

Proposition 2.1. Let $W: A \rightarrow R$ be an elliptic net. Then $W(-z)=-W(z)$ for all $z \in A$.

The purpose of this section is to find a finite subset $0 \notin J \subset I$ such that the localisation $\mathscr{W}_{I}\left[T_{i}^{-1}\right]_{i \in J}$ is finitely generated as a $\mathbb{Z}$-algebra, and to give the generators. (The localisation is not the trivial ring $(1=0)$ by the existence of a homomorphism from it to $\mathbb{Q}$ given by Example 1.2, where one uses part (3) with $W^{\prime}=W_{i d}$ of part (2).) From this we show that every $T_{i}$ can be expressed as a Laurent polynomial in integer coefficients in a finite number of terms $T_{j}$. This implies that any elliptic net which does not take zero values at the $T_{j}$ is entirely determined by those values.

To illustrate, consider the rank-one case, which is essentially a result of Morgan Ward.

Theorem 2.2 [Ward 1948, Theorem 4.1]. The ring $\mathcal{W}_{\mathbb{Z}}\left[T_{1}^{-1}, T_{2}^{-1}\right]$ is generated as $a \mathbb{Z}$-algebra by the six elements

$$
T_{1}, \quad T_{1}^{-1}, \quad T_{2}, \quad T_{2}^{-1}, \quad T_{3}, \quad T_{4} .
$$

Furthermore, each $T_{i}$ is expressible as a $\mathbb{Z}$-coefficient polynomial in

$$
T_{1}, \quad T_{1}^{-1}, \quad T_{2}, \quad T_{3}, \quad T_{4} T_{2}^{-1} .
$$

In particular, let $W: \mathbb{Z} \rightarrow \mathbb{Q}$ be an elliptic net. If $W(1)=1, W(2) \neq 0, W(i)$ is an integer for $i=2,3,4$, and $W(2)$ divides $W(4)$, then the elliptic net consists entirely of integers.

Proof. Recall that $T_{-n}=-T_{n}$, so it suffices to prove the first two statements for positive $n$. Taking $(p, q, r, s)=(n+1, n, 1,0)$ and $(n+1, n-1,1,0)$ respectively, in $W_{I}$ we have

$$
\begin{gathered}
T_{2 n+1} T_{1}^{3}+T_{n-1} T_{n+1}^{3}+T_{n+2} T_{-n} T_{n}^{2}=0, \\
T_{2 n} T_{2} T_{1}^{2}+T_{n} T_{n-2} T_{n+1}^{2}+T_{n+2} T_{-n} T_{n-1}^{2}=0 .
\end{gathered}
$$

The equations (6) and (7) prove the first statement by induction. The base case consists of $0 \leq n \leq 4$; for $n>4$, we have $2 n>n+2$. 
For even $i$, it can be shown by induction on (7) that $T_{i}$ is expressible as a $\mathbb{Z}$ coefficient polynomial in $T_{1}, T_{1}^{-1}, T_{2}, T_{2}^{-1}, T_{3}$, and $T_{4}$ in such a way that the combined degree of $T_{2}$ and $T_{4}$ in each monomial is positive. For $i=2,4$ this is clear. To complete the induction in general, observe that in (7), each of the rightmost two terms is divisible by at least two $T_{k}$ where $k$ is even and $k<2 n$.

For even $i$, the second statement of the theorem concerning the expressibility of all $T_{i}$ in terms of $T_{1}, T_{1}^{-1}, T_{2}, T_{3}$ and $T_{4} T_{2}^{-1}$ follows from the observation of the previous paragraph. The statement also holds for $i=1,3$. Consequently, it holds for odd $i$ by induction on (6).

Proofs by induction. The inductive proofs in this section will be based on the following definitions. Consider finite sets $S, J \subset I$ where $0, i \notin S \cup J$. We say that an index $i \in I$ is $S$-integrally implied by $J$ if there exists a $\mathbb{Z}$-coefficient monomial $P\left(T_{S}\right)$ (in variables indexed by $S$ ) and $\mathbb{Z}$-coefficient polynomial $Q\left(T_{j}\right)$ (in variables indexed by $J$ ) such that

$$
T_{i} P\left(T_{s}\right)=Q\left(T_{j}\right)
$$

in $W_{I}$. A set $K \subset I$ is $S$-integrally implied by the set $J$ if every index in $K$ is $S$-integrally implied by $J$.

As an example (see Proposition 2.1 and the paragraph which precedes it), $-i$ is $S$-integrally implied by any $J$ containing $i$ (for any $S$ ). In what follows, this fact will often be used tacitly.

A set $B \subset I$ is an $S$-integral baseset for $\mathscr{W}_{I}$ if all of $I$ is $S$-integrally implied by $B$. If $B \subset I$ is an $S$-integral baseset, then each $T_{i}$ can be expressed as a polynomial with integer coefficients in the set of variables $\left\{T_{b}\right\}_{b \in B} \cup\left\{T_{s}^{-1}\right\}_{s \in S}$ (when considered in the appropriate localisation).

It is straightforward to verify that if $i$ is $S$-integrally implied by $J$ and every $j \in J$ is $S$-integrally implied by $J^{\prime}$, then $i$ is $S$-integrally implied by $J^{\prime}$. To show that $B$ is an $S$-integral baseset for $I$, the proofs in this section show the following: for each index $i \in I$, there is a finite sequence $J_{0} \subset J_{1} \subset \cdots \subset J_{n}$ such that $B=J_{0}$, $i \in J_{n}$ and for each $1 \leq k \leq n, J_{k}$ is $S$-integrally implied by $J_{k-1}$. At each stage, we show that each index of $J_{i}$ is $S$-integrally implied by $J_{i-1}$. Recall that implication is simply the existence of an relation of the form (8), and in fact we simply give a relevant element of the form (5).

These elements are cumbersome to write out. For example, taking in the case $n=3, \boldsymbol{p}=(1,0,0), \boldsymbol{q}=(0,1,0), \boldsymbol{r}=(0,0,1), \boldsymbol{s}=(0,0,0)$, we obtain

$T_{(1,1,0)} T_{(1,-1,0)} T_{(0,0,1)} T_{(0,0,1)}$

$$
\begin{aligned}
+T_{(0,1,1)} T_{(0,1,-1)} T_{(1,0,0)} & T_{(1,0,0)} \\
& +T_{(1,0,1)} T_{(-1,0,1)} T_{(0,1,0)} T_{(0,1,0)} .
\end{aligned}
$$


For this information, let us instead use the more convenient notation

$$
\begin{array}{rrrr}
1 & 0 & 0 & 0 \\
0 & 1 & 0 & 0 \\
0 & 0 & 1 & 0
\end{array}\left[\begin{array}{rrrr|rrrr|rrrr}
1 & 1 & 0 & 0 & 0 & 0 & 1 & 1 & 1 & -1 & 0 & 0 \\
1 & -1 & 0 & 0 & 1 & 1 & 0 & 0 & 0 & 0 & 1 & 1 \\
0 & 0 & 1 & 1 & 1 & -1 & 0 & 0 & 1 & 1 & 0 & 0
\end{array}\right] \text {. }
$$

In this notation, the columns to the left of the brackets correspond to the columns of $p, q, r$ and $s$, while the indices of the terms of the recurrence appear as the columns within the brackets.

To demonstrate that an index $i$ is ( $S$-integrally) implied by a set of indices $J$, it suffices to write down an appropriate such array. Notice that any array of the form (9) is a recurrence if each row is a recurrence. Therefore we may construct examples row by row.

The following definition will be useful for ordering inductions.

Definition 2.3. Let

$$
N(\boldsymbol{v})=\max _{i=1, \ldots, n}\left|v_{i}\right|
$$

be the sup-norm of the vector $\boldsymbol{v}$.

Basesets for rank 2. For the rank-two case, we require a lemma.

Lemma 2.4. The ring $\mathscr{W}_{\mathbb{Z}^{2}}\left[T_{(1,0)}^{-1}, T_{(0,1)}^{-1}, T_{(1,1)}^{-1}\right]$ is generated as a $\mathbb{Z}$-algebra by the elements

$$
\left\{T_{v}: N(v) \leq 4\right\} \cup\left\{T_{(1,0)}^{-1}, T_{(0,1)}^{-1}, T_{(1,1)}^{-1}\right\} .
$$

Proof. Let $S=\{(1,0),(0,1),(1,1)\}$ and $B=\left\{\boldsymbol{v} \in \mathbb{Z}^{2}: N(\boldsymbol{v}) \leq 4\right\}$. This proof proceeds by induction on the sup-norm. Trivially, any $\boldsymbol{v}$ with $N(\boldsymbol{v}) \leq 4$ is $S$ integrally implied by $B$. Let $N_{0}>4$ and suppose that all terms with indices with sup-norm less than $N_{0}$ are $S$-integrally implied by $B$. Call the set of such indices $K_{N_{0}}$. Suppose $\boldsymbol{v}$ is an index of sup-norm $N_{0}$. We construct a recurrence demonstrating that $\boldsymbol{v}$ is $S$-integrally implied by $K_{N_{0}}$ row by row. For $i=1,2$, define $w_{i}=\left\lceil v_{i} / 2\right\rceil$.

Case I: $v$ has one odd entry and one even entry. For the odd entry, we use the row

$$
w_{i} \quad w_{i}-1 \quad 0 \quad 0\left[\begin{array}{lllllllllllll}
v_{i} & 1 & 0 & 0 & \mid w_{i}-1 & w_{i}-1 & w_{i} & w_{i} \mid & w_{i} & -w_{i} & w_{i}-1 & w_{i}-1
\end{array}\right]
$$

For the even entry, we use the row

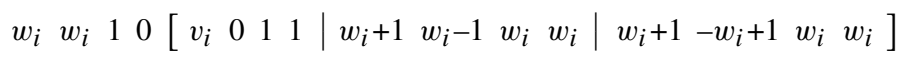

Case II: $v$ has two odd entries. Use the rows

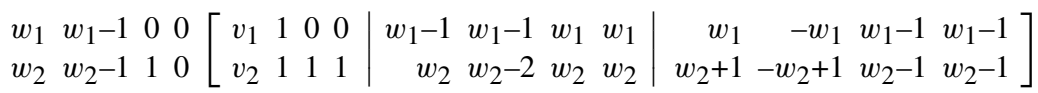


Case III: $v$ has two even entries. Use the rows

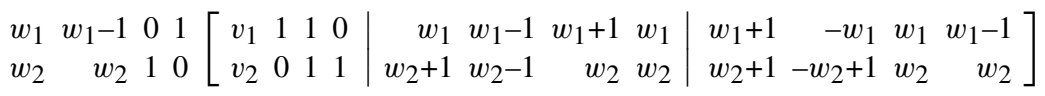

For even $v_{i}$, either $\left|v_{i}\right| \leq 2$ or $\left|v_{i}\right|>3$. In the former case, $\left|w_{i}\right|+1 \leq 2<N_{0}$. In the latter case, we have $\left|w_{i}\right|+1 \leq\left(\left|v_{i}\right|+2\right) / 2<\left|v_{i}\right| \leq N_{0}$. For odd $\boldsymbol{v}_{i}$, either $\left|v_{i}\right| \leq 3$ or $\left|v_{i}\right|>4$. In the former case $\left|w_{i}\right|+2 \leq 4<N_{0}$. In the latter case, we have $\left|w_{i}\right|+2 \leq\left(\left|v_{i}\right|+5\right) / 2<\left|v_{i}\right| \leq N_{0}$.

Therefore all the vectors in the recurrence have sup-norm less than $N_{0}$ with the exception of $\boldsymbol{v}$. In the monomial of $\boldsymbol{v}$ in the recurrence, the other indices are $(1,0)$, $(0,1)$ or $(1,1)$. This demonstrates that $\boldsymbol{v}$ is $S$-integrally implied by $K_{N_{0}}$ and hence by $B$.

Theorem 2.5. The ring $\mathscr{W}_{\mathbb{Z}^{2}}\left[T_{(1,1)}^{-1}, T_{(1,0)}^{-1}, T_{(0,1)}^{-1}\right]$ is generated as a $\mathbb{Z}$-algebra by the eleven elements

$$
T_{(1,1)}, T_{(1,0)}, T_{(0,1)}, T_{(1,1)}^{-1}, T_{(1,0)}^{-1}, T_{(0,1)}^{-1}, T_{(2,1)}, T_{(1,2)}, T_{(2,0)}, T_{(0,2)}, T_{(2,2)},
$$

and the following identities hold:

$$
\begin{aligned}
T_{(1,-1)} T_{(1,1)}^{3} & =T_{(1,0)}^{3} T_{(1,2)}-T_{(0,1)}^{3} T_{(2,1)}, \\
T_{(2,2)} T_{(1,-1)} T_{(1,0)} T_{(0,1)} & =T_{(1,1)}\left(T_{(0,2)} T_{(2,1)} T_{(1,0)}-T_{(0,1)} T_{(2,0)} T_{(1,2)}\right) .
\end{aligned}
$$

In particular, if $W: \mathbb{Z}^{2} \rightarrow \mathbb{Q}$ is an elliptic net for which

(a) $W(1,0)=W(0,1)=W(1,1)=1$,

(b) $W(2,0), W(0,2), W(1,2) \neq W(2,1)$ are integers, and

(c) $W(1,2)-W(2,1)$ divides $W(0,2) W(2,1)-W(2,0) W(1,2)$,

then all terms of the elliptic net are determined by these seven values and are integers.

Proof. The first and second stated identities are the recurrences

$$
\begin{aligned}
& \begin{array}{rrrr}
1 & 0 & 1 & 0 \\
0 & 1 & 1 & 0
\end{array}\left[\begin{array}{rrrr|rrrr|rrrr}
1 & 1 & 1 & 1 & 1 & -1 & 1 & 1 & 2 & 0 & 0 & 0 \\
1 & -1 & 1 & 1 & 2 & 0 & 0 & 0 & 1 & 1 & 1 & 1
\end{array}\right], \\
& \begin{array}{rrrr}
1 & 1 & -1 & 0 \\
1 & 2 & 1 & -1
\end{array}\left[\begin{array}{rrrr|rrrr|rrrr}
2 & 0 & -1 & -1 & 0 & 2 & 1 & 1 & 0 & -2 & 1 & 1 \\
2 & -1 & 0 & 1 & 2 & 1 & 0 & 1 & 1 & 0 & 1 & 2
\end{array}\right] .
\end{aligned}
$$

Let $S=\{(1,0),(0,1),(1,1)\}$, and $B=\left\{\boldsymbol{v} \in \mathbb{Z}^{2}: N(\boldsymbol{v}) \leq 4\right\}$. By Lemma 2.4, it suffices to show that $B$ is $S$-integrally implied by the set

$$
\{(1,0),(0,1),(1,1),(2,0),(0,2),(2,1),(1,2),(2,2)\} .
$$

We list the relevant recurrences in order. As each index is implied, it may be used to imply later indices. It is assumed that as $(a, b)$ is implied, so is $(-a,-b)$. To begin, the index $(1,-1)$ is implied by $(10)$. We then write 


$$
\begin{aligned}
& (2,-1): \quad \begin{array}{rrrr}
-1 & 0 & 1 & 1 \\
1 & 1 & 0 & 0
\end{array}\left[\begin{array}{rrrr|rrrr|rrrr}
0 & -1 & 2 & 1 & 2 & -1 & 0 & -1 & 1 & 2 & 1 & 0 \\
2 & 0 & 0 & 0 & 1 & 1 & 1 & 1 & 1 & -1 & 1 & 1
\end{array}\right], \\
& (-1,2): \quad \begin{array}{rrrrr}
0 & -1 & -1 & 0
\end{array} \quad\left[\begin{array}{rrrr|rrrr|rrrr}
-1 & 1 & -1 & -1 & -2 & 0 & 0 & 0 & -1 & -1 & -1 & -1 \\
2 & 0 & 0 & 0 & 1 & 1 & 1 & 1 & 1 & -1 & 1 & 1
\end{array}\right] \text {, } \\
& (2,-2): \quad \begin{array}{rrrl}
1 & 1 & -1 & 0
\end{array} \quad\left[\begin{array}{rrrr|rrrr|rrrr}
2 & 0 & -1 & -1 & 0 & 2 & 1 & 1 & 0 & -2 & 1 & 1 \\
-2 & 1 & 0 & -1 & -2 & -1 & 0 & -1 & -1 & 0 & -1 & -2
\end{array}\right] \text {. }
\end{aligned}
$$

At this point we have implied all indices of sup-norm at most 2. Next we have

$$
\begin{aligned}
& (3,0): \quad \begin{array}{lllll}
2 & 1 & 0 & 0 \\
0 & 0 & 1 & 0
\end{array}\left[\begin{array}{llll|lrll|rrrr}
3 & 1 & 0 & 0 & 1 & 1 & 2 & 2 & 2 & -2 & 1 & 1 \\
0 & 0 & 1 & 1 & 1 & -1 & 0 & 0 & 1 & 1 & 0 & 0
\end{array}\right], \\
& (3,1): \quad \begin{array}{lllll}
2 & 1 & 0 & 0 \\
& 1 & 0 & 1 & 0
\end{array}\left[\begin{array}{llll|lrll|rrrr}
3 & 1 & 0 & 0 & 1 & 1 & 2 & 2 & 2 & -2 & 1 & 1 \\
1 & 1 & 1 & 1 & 1 & -1 & 1 & 1 & 2 & 0 & 0 & 0
\end{array}\right] \text {, } \\
& (3,2): \quad \begin{array}{lllll}
2 & 1 & 0 & 0 \\
& 1 & 1 & 1 & 0
\end{array}\left[\begin{array}{llll|llll|rrrr}
3 & 1 & 0 & 0 & 1 & 1 & 2 & 2 & 2 & -2 & 1 & 1 \\
2 & 0 & 1 & 1 & 2 & 0 & 1 & 1 & 2 & 0 & 1 & 1
\end{array}\right] \text {, } \\
& (3,3): \quad \begin{array}{lllll}
2 & 1 & 1 & 0 \\
& 2 & 1 & 0 & 0
\end{array}\left[\begin{array}{llll|llll|llll}
3 & 1 & 1 & 1 & 2 & 0 & 2 & 2 & 3 & -1 & 1 & 1 \\
3 & 1 & 0 & 0 & 1 & 1 & 2 & 2 & 2 & -2 & 1 & 1
\end{array}\right] \text {. }
\end{aligned}
$$

Simply by switching top rows with bottom rows, we similarly imply $(0,3),(1,3)$, and $(2,3)$. And by putting negatives on the second row of (11), we imply the index $(3,-2)$ (and $(-2,3)$ by switching top and bottom). Next,

$$
\begin{aligned}
& (3,-1): \quad \begin{array}{rrrr}
2 & 1 & 0 & 0 \\
-1 & -1 & -2 & 2
\end{array}\left[\begin{array}{rrrr|rrrr|rrrr}
3 & 1 & 0 & 0 & 1 & 1 & 2 & 2 & 2 & -2 & 1 & 1 \\
-1 & 1 & 1 & -1 & -1 & -1 & 1 & -1 & 0 & 0 & 0 & -2
\end{array}\right], \\
& (3,-3): \quad \begin{array}{rrrl}
1 & 2 & 1 & 0 \\
-2 & -1 & 0 & 0
\end{array}\left[\begin{array}{rrrr|rrrr|rrrr}
3 & -1 & 1 & 1 & 3 & 1 & 1 & 1 & 2 & 0 & 2 & 2 \\
-3 & -1 & 0 & 0 & -1 & -1 & -2 & -2 & -2 & 2 & -1 & -1
\end{array}\right] \text {. }
\end{aligned}
$$

Again by switching top and bottom we get $(-1,3)$. We have now implied all indices with sup-norm at most 3 . We continue with

$$
\begin{aligned}
& (4,0): \quad \begin{array}{lllll}
2 & 1 & 0 & 1 \\
0 & 0 & 1 & 0
\end{array}\left[\begin{array}{llll|lrll|rrrr}
4 & 1 & 1 & 0 & 2 & 1 & 3 & 2 & 3 & -2 & 2 & 1 \\
0 & 0 & 1 & 1 & 1 & -1 & 0 & 0 & 1 & 1 & 0 & 0
\end{array}\right], \\
& (4,1): \quad \begin{array}{lllll}
3 & 2 & 1 & -1 \\
0 & 0 & 0 & 1
\end{array}\left[\begin{array}{llll|llll|rrrr}
4 & 1 & 0 & 1 & 2 & 1 & 2 & 3 & 3 & -2 & 1 & 1 \\
1 & 0 & 1 & 0 & 1 & 0 & 1 & 0 & 1 & 0 & 1 & 0
\end{array}\right] \text {, } \\
& \left.(4,2): \quad \begin{array}{lllll}
3 & 2 & 1 & -1
\end{array} \quad \begin{array}{llll|llll|rrrr}
4 & 1 & 0 & 1 & 2 & 1 & 2 & 3 & 3 & -2 & 1 & 2 \\
2 & 0 & 1 & 1 & 2 & 0 & 1 & 1 & 2 & 0 & 1 & 1
\end{array}\right] \text {, } \\
& (4,3): \quad \begin{array}{lllll}
2 & 2 & 1 & 0 \\
& 2 & 1 & 0 & 0
\end{array}\left[\begin{array}{llll|llll|llll}
4 & 0 & 1 & 1 & 3 & 1 & 2 & 2 & 3 & -1 & 2 & 2 \\
3 & 1 & 0 & 0 & 1 & 1 & 2 & 2 & 2 & -2 & 1 & 1
\end{array}\right] \text {, } \\
& \left.(4,4): \quad \begin{array}{llllll}
3 & 2 & 1 & -1
\end{array} \quad \begin{array}{llll|llll|llll}
4 & 1 & 0 & 1 & 2 & 1 & 2 & 3 & 3 & -2 & 1 & 2 \\
4 & 0 & 1 & 1 & 3 & 1 & 2 & 2 & 3 & -1 & 2 & 2
\end{array}\right] .
\end{aligned}
$$

Again by switching top rows with bottom rows, we similarly imply $(0,4),(1,4)$, $(2,4)$ and $(3,4)$. And by putting negatives on the second rows, we imply the indices $(4,-1),(-1,4),(4,-3)$ and $(-3,4)$. There remains to consider the 
indices

$$
\begin{aligned}
& (4,-2): \quad \begin{array}{rrrl}
2 & 1 & -1 & 1 \\
-1 & -1 & -1 & 0
\end{array}\left[\begin{array}{rrrr|rrrr|rrrr}
4 & 1 & 0 & -1 & 1 & 2 & 3 & 2 & 2 & -3 & 2 & 1 \\
-2 & 0 & -1 & -1 & -2 & 0 & -1 & -1 & -2 & 0 & -1 & -1
\end{array}\right], \\
& (4,-4): \quad \begin{array}{rrrr}
2 & 1 & -1 & 1 \\
-2 & -2 & -1 & 0
\end{array}\left[\begin{array}{rrrr|rrrr|rrrr}
4 & 1 & 0 & -1 & 1 & 2 & 3 & 2 & 2 & -3 & 2 & 1 \\
-4 & 0 & -1 & -1 & -3 & -1 & -2 & -2 & -3 & 1 & -2 & -2
\end{array}\right] \text {. }
\end{aligned}
$$

By switching rows, we imply $(-2,4)$. We have now demonstrated the calculation of all terms of index with sup-norm at most 4 . The second part of the statement follows immediately from the first.

Basesets for ranks $\boldsymbol{n} \geq 3$. Let $\boldsymbol{e}_{i}$ denote the standard basis vectors.

Lemma 2.6. Define subsets of $\mathbb{Z}^{3}$ by

$$
\begin{aligned}
& L_{2}=\left\{\boldsymbol{e}_{i}\right\}_{i} \cup\left\{\boldsymbol{e}_{i} \pm \boldsymbol{e}_{j}\right\}_{i \neq j} \cup\left\{2 \boldsymbol{e}_{i}\right\}_{i}, \\
& L_{2}^{\prime}=\left\{a_{i} \boldsymbol{e}_{i}+a_{j} \boldsymbol{e}_{j}: a_{i} \in \mathbb{Z}, 1 \leq i \leq j \leq 3\right\} .
\end{aligned}
$$

Then all indices $\boldsymbol{v} \in \mathbb{Z}^{3}$ with $N(\boldsymbol{v}) \leq 2$ are $L_{2}$-integrally implied by $L_{2}^{\prime}$.

Proof. We make use of the recurrences

$$
\begin{aligned}
& \begin{array}{rrrr}
1 & 1 & 0 & -1 \\
0 & 0 & -1 & 1 \\
1 & 0 & 1 & 0
\end{array}\left[\begin{array}{rrrr|rrrr|rrrr}
1 & 0 & -1 & 0 & 0 & 1 & 0 & 1 & 0 & -1 & 0 & 1 \\
1 & 0 & 0 & -1 & 0 & 1 & 1 & 0 & 0 & -1 & 1 & 0 \\
1 & 1 & 1 & 1 & 1 & -1 & 1 & 1 & 2 & 0 & 0 & 0
\end{array}\right], \\
& \begin{array}{rrrr}
0 & 0 & 1 & -1 \\
1 & 1 & 0 & -1 \\
0 & 1 & 1 & 0
\end{array}\left[\begin{array}{rrrr|rrrr|rrrr}
-1 & 0 & 0 & 1 & 0 & -1 & -1 & 0 & 0 & 1 & -1 & 0 \\
1 & 0 & -1 & 0 & 0 & 1 & 0 & 1 & 0 & -1 & 0 & 1 \\
1 & -1 & 1 & 1 & 2 & 0 & 0 & 0 & 1 & 1 & 1 & 1
\end{array}\right] \text {, } \\
& \begin{array}{rrrr}
1 & 0 & 1 & 0 \\
0 & 0 & 0 & 1 \\
1 & 1 & 0 & -1
\end{array}\left[\begin{array}{rrrr|rrrr|rrrr}
1 & 1 & 1 & 1 & 1 & -1 & 1 & 1 & 2 & 0 & 0 & 0 \\
1 & 0 & 1 & 0 & 1 & 0 & 1 & 0 & 1 & 0 & 1 & 0 \\
1 & 0 & -1 & 0 & 0 & 1 & 0 & 1 & 0 & -1 & 0 & 1
\end{array}\right] \text {. }
\end{aligned}
$$

Permute the rows of (12) by the cyclic permutations (123) and (132), calling the results (12)' and (12)" respectively; for example, the rightmost column of (12)' is $(0,1,0)$. Do the same for (13) and (14).

Consider the equation obtained by the combination

$$
\begin{aligned}
&(12) \times T_{(1,1,1)} T_{(1,0,0)}^{2} T_{(1,-1,0)} T_{(0,1,0)}^{2}+(12)^{\prime} \times T_{(1,1,1)} T_{(1,0,0)} T_{(0,1,-1)} T_{(0,1,0)}^{2} T_{(0,0,1)} \\
&+(14) \times T_{(1,-1,0)} T_{(0,1,0)}^{2} T_{(0,1,1)} T_{(0,0,1)} T_{(1,0,1)}^{2}+(14)^{\prime} \times T_{(0,1,-1)} T_{(1,0,0)}^{2} T_{(0,0,1)} T_{(1,0,1)} T_{(1,1,0)} \\
&+(13) \times T_{(1,1,1)} T_{(1,0,0)}^{2} T_{(0,1,0)}^{2} T_{(1,1,0)}+(13)^{\prime} \times T_{(1,1,1)} T_{(0,1,0)}^{2} T_{(1,0,0)} T_{(0,1,1)} T_{(0,0,1)} \\
&+(13)^{\prime \prime} \times T_{(1,1,1)} T_{(1,0,0)}^{2} T_{(1,0,1)} T_{(0,1,0)} T_{(0,0,1)} .
\end{aligned}
$$

The result has the form $a T_{(1,1,1)}+b=0$, where $a$ and $b$ are polynomials in $T_{v}$ where every $\boldsymbol{v}$ has at least one zero coordinate. In particular,

$$
a=T_{(1,0,0)}^{3} T_{(0,1,0)} T_{(0,0,1)}^{2} T_{(1,0,1)} T_{(0,2,0)} T_{(1,0,-1)} .
$$


Thus $T_{(1,1,1)}$ is $L_{2}$-integrally implied by $L_{2}^{\prime}$. To imply the terms $T_{(-1,1,1)}, T_{(1,-1,1)}$, and $T_{(1,1,-1)}$, use $(12),(12)^{\prime}$, and $(12)^{\prime \prime}$. This covers all terms of sup-norm at most 1 .

We have the following recurrence:

$\begin{array}{rrrr}0 & 0 & 0 & 1 \\ 0 & 0 & -1 & 1 \\ 2 & 1 & 1 & -1 \\ 0 & 1 & 0 & 1 \\ 1 & 1 & 0 & 0\end{array}\left[\begin{array}{rrrr|rrrr|rrrr}1 & 0 & 1 & 0 & 1 & 0 & 1 & 0 & 1 & 0 & 1 & 0 \\ 1 & 0 & 0 & -1 & 0 & 1 & 1 & 0 & 0 & -1 & 1 & 0 \\ 2 & 1 & 0 & 1 & 1 & 0 & 1 & 2 & 2 & -1 & 0 & 1 \\ 2 & -1 & 1 & 0 & 2 & 1 & 1 & 0 & 1 & 0 & 2 & 1 \\ 2 & 0 & 0 & 0 & 1 & 1 & 1 & 1 & 1 & -1 & 1 & 1\end{array}\right]$.

If $\boldsymbol{v}$ has exactly one coordinate of value \pm 2 (the rest \pm 1 ), then we imply $\boldsymbol{v}$ by taking the first three rows in the recurrence above (possibly taking negatives and permutations of rows as necessary). If $\boldsymbol{v}$ has exactly two \pm 2 's, use the middle three rows in the same way. If $v$ has exactly three \pm 2 's, use the last three rows (this relies on the previous cases).

Remark 2.7. The four equations (12), (12)', (12)" and (13) in the four unknowns $T_{(1,1,1)}, T_{(-1,1,1)}, T_{(1,-1,1)}$ and $T_{(1,1,-1)}$, are linear with coefficients consisting of monomials in $T_{v}$ where $v$ has at least one zero coordinate. The determinant of the system is

$$
2 T_{(1,0,0)} T_{(0,1,0)} T_{(0,0,1)}^{2} T_{(1,1,0)} T_{(1,0,1)}^{2} T_{(0,1,1)}^{2} T_{(1,-1,0)} T_{(1,0,-1)} T_{(0,1,-1)} .
$$

This observation is useful for calculations where 2 is invertible.

Theorem 2.8. Let $n \geq 2$. For each $\ell$ in the set

$$
L=\{0,1\}^{n} \backslash\{(0,0, \ldots, 0),(1,1, \ldots, 1)\},
$$

choose a vector $\boldsymbol{x}_{\ell}$ having $N\left(\boldsymbol{x}_{\ell}\right)=1$ and having nonzero entries exactly where $\ell$ does. Let $G_{n}=\left\{\boldsymbol{x}_{\ell}\right\}_{\ell \in L}$. Let

$$
\begin{aligned}
& H_{n}=G_{n} \cup\left\{\boldsymbol{e}_{i}\right\} \cup\left\{\boldsymbol{e}_{i} \pm \boldsymbol{e}_{j}, i \neq j\right\} \cup\left\{2 \boldsymbol{e}_{i}\right\}, \\
& H_{n}^{\prime}=H_{n} \cup\left\{2 \boldsymbol{e}_{i}+\boldsymbol{e}_{j}, i \neq j\right\} .
\end{aligned}
$$

Then $\mathbb{Z}^{n}$ is $H_{n}$-integrally implied by $H_{n}^{\prime}$.

Proof. The proof is by induction on $n$. The base case is $n=2$, which is a consequence of Theorem 2.5 .

Fixing any $1 \leq i \leq n$, we can identify $H_{n-1}$ with a subset of $H_{n}$ (and $H_{n-1}^{\prime}$ with a subset of $H_{n}^{\prime}$ ) by adding a zero between the $(i-1)$-th and $i$-th positions of each vector of $H_{n-1}$ (or $H_{n-1}^{\prime}$ ). By this identification and by the inductive hypothesis (for $n-1$ ), any $\boldsymbol{v} \in \mathbb{Z}^{n}$ with a zero in the $i$-th position is $H_{n}$-integrally implied by $H_{n}^{\prime}$. Therefore it suffices to imply those $\boldsymbol{v} \in \mathbb{Z}^{n}$ having no zero coordinate.

The inductive step is itself an induction on the sup-norm of $\boldsymbol{v}$. The base cases are $N(\boldsymbol{v})=1$ and $N(\boldsymbol{v})=2$. Both of these for $n=3$ are provided by Lemma 2.6, 
so for the base cases, we may assume $n \geq 4$. To imply $\boldsymbol{v}$, we construct a recurrence row by row, so that the first column is exactly $\boldsymbol{v}$. For the first three rows, use the following, multiplied by -1 as necessary.

$$
\begin{array}{llll}
1 & 0 & 0 & 0 \\
0 & 1 & 0 & 0 \\
0 & 0 & 0 & 1
\end{array}\left[\begin{array}{rrrr|rrrr|rrrr}
1 & 1 & 0 & 0 & 0 & 0 & 1 & 1 & 1 & -1 & 0 & 0 \\
1 & -1 & 0 & 0 & 1 & 1 & 0 & 0 & 0 & 0 & 1 & 1 \\
1 & 0 & 1 & 0 & 1 & 0 & 1 & 0 & 1 & 0 & 1 & 0
\end{array}\right] .
$$

For all subsequent rows, use one of the following two recurrences (shown together in an array), multiplied by -1 as appropriate:

$$
\begin{array}{rrrr}
1 & 1 & 1 & -1 \\
0 & 0 & -1 & 1
\end{array}\left[\begin{array}{rrrr|rrrr|rrrr}
1 & 0 & 0 & 1 & 1 & 0 & 0 & 1 & 1 & 0 & 0 & 1 \\
1 & 0 & 0 & -1 & 0 & 1 & 1 & 0 & 0 & -1 & 1 & 0
\end{array}\right] .
$$

For each row, the choice between the two possibilities can be made in such a way that the fourth column of the recurrence lies in $G_{n}$. Columns 2 and 4 have at most two nonzero entries (which are \pm 1 ) and so are in $H_{n}$. The other columns, numbered 5 though 12, have at least one zero entry, and so are already implied by the inductive step. This completes the case $N(\boldsymbol{v})=1$.

For the remainder of the proof, we will repeatedly use the following recurrences. Let $w_{i}=\left\lceil v_{i} / 2\right\rceil$. If $v_{i}$ is even, we call the recurrences shown in the following array (E1) through (E4):

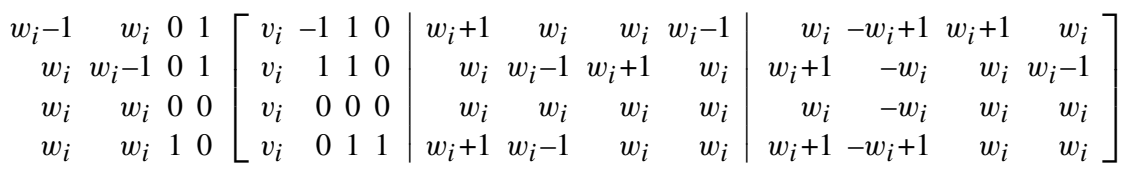

If $v_{i}$ is odd, we call the following recurrences (O1) through (O5).

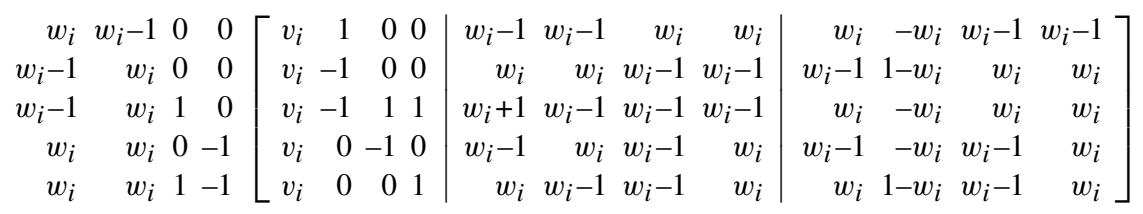

The second base case is $N(\boldsymbol{v})=2\left(n \geq 4\right.$ still). Since we may assume $v_{i} \neq 0$ (this is covered by previous cases in the induction on $n$ ), the other $v_{i}$ have $\left|v_{i}\right|= \pm 1$. There are three cases:

Case I: $\boldsymbol{v}$ has at least three odd $v_{i}$. Use for the first three odd $v_{i}$ the recurrences $(\mathrm{O} 1),(\mathrm{O} 4)$ and $(\mathrm{O} 5)$ respectively. Use (E3) for all the even $v_{i}$. In this case, all the columns besides the first contain only digits 0 and \pm 1 and so were implied in the case $N(\boldsymbol{v})=1$. Columns 2,3 , and 4 contain only one nonzero term each, and so are in $H_{n}$. 
Case II: $v$ has one or two odd $v_{i}$. Use (O3) for one odd coordinate and (O1) for the other (if it exists). Use (E3) for all even coordinates. Then, columns 2-4 contain one or two nonzero entries, and columns 5-12 may contain at most one \pm 2 ; but such a column was implied in the Case I.

Case III: $v$ has no odd $v_{i}$. Use (E1) and (E4) for the first two rows, and (E3) for all others. Columns 2-4 contain one or two nonzero entries and 5-12 at most two \pm 2 's; but such a column was implied in Case I or II.

This completes the $N(\boldsymbol{v})=2$ base case.

Now suppose $N(\boldsymbol{v})=N_{0} \geq 3$ and $n \geq 3$. This is the inductive step; we will assume we have implied all indices of sup-norm less than $N_{0}$. As before, $v_{i} \neq 0$. For $\left|v_{i}\right|=3,(\mathrm{O} 1),(\mathrm{O} 2),(\mathrm{O} 4)$, and $(\mathrm{O} 5)$ have entries less than $N_{0}$ in columns 5-12. For $1 \leq\left|v_{i}\right| \leq 2$, and $3<\left|v_{i}\right| \leq N_{0}$, all applicable recurrences have entries less than $N_{0}$ in those columns. We have two cases:

Case I: $v$ has at least one even entry. Use (E4) for the first even coordinate, and choose from (E1) and (E2) for the second even coordinate (if it exists). We use (E3) for all other even coordinates. We will use (O1) or (O2) for all odd entries (and make the choice between (E1) and (E2) above) in such a way that the second column is in $G_{n}$.

Case II: $v$ has no even entry. Use (O4) and (O5) for the first two odd coordinates, and (O1) or (O2) for all others, according so that the second column is an element of $G_{n}$.

\section{Net polynomials over $\mathbb{C}$}

Fix an elliptic curve $E$ defined over $\mathbb{C}$. Our purpose is to define rational functions $\Omega_{v}: E^{n} \rightarrow \mathbb{C}$ for all $\boldsymbol{v} \in \mathbb{Z}^{n}$ such that for each $\boldsymbol{P} \in E^{n}$, the map

$$
W_{E, \boldsymbol{P}}: \mathbb{Z}^{n} \rightarrow \mathbb{C}, \quad \boldsymbol{v} \mapsto \Omega_{\boldsymbol{v}}(\boldsymbol{P})
$$

is an elliptic net. In this section we associate a lattice $\Lambda \subset \mathbb{C}$ to the elliptic curve $E$ and consider the complex uniformization $\mathbb{C} / \Lambda$.

Elliptic functions over $\mathbb{C}$. For a complex lattice $\Lambda$, let $\eta: \Lambda \rightarrow \mathbb{C}$ be the quasiperiod homomorphism, and define a quadratic form $\lambda: \Lambda \rightarrow\{ \pm 1\}$ by

$$
\lambda(\omega)=\left\{\begin{aligned}
1 & \text { if } \omega \in 2 \Lambda, \\
-1 & \text { if } \omega \notin 2 \Lambda .
\end{aligned}\right.
$$

Recall that the Weierstrass sigma function $\sigma: \mathbb{C} / \Lambda \rightarrow \mathbb{C}$ satisfies the following transformation formula for all $z \in \mathbb{C}$ and $\omega \in \Lambda$ :

$$
\sigma(z+\omega ; \Lambda)=\lambda(\omega) e^{\eta(\omega)\left(z+\frac{1}{2} \omega\right)} \sigma(z ; \Lambda) .
$$


Definition 3.1. Fix a lattice $\Lambda \in \mathbb{C}$ corresponding to an elliptic curve $E$. For $\boldsymbol{v}=\left(v_{1}, \ldots, v_{n}\right) \in \mathbb{Z}^{n}$, define a function $\Omega_{\boldsymbol{v}}$ on $\mathbb{C}^{n}$ in variables $\boldsymbol{z}=\left(z_{1}, \ldots, z_{n}\right)$ as follows:

$$
\Omega_{v}(z ; \Lambda)=\frac{\sigma\left(v_{1} z_{1}+\cdots+v_{n} z_{n} ; \Lambda\right)}{\prod_{i=1}^{n} \sigma\left(z_{i} ; \Lambda\right)^{2 v_{i}^{2}-\sum_{j=1}^{n} v_{i} v_{j}} \prod_{1 \leq i<j \leq n} \sigma\left(z_{i}+z_{j} ; \Lambda\right)^{v_{i} v_{j}}} .
$$

(If $\boldsymbol{v}=\mathbf{0}$, we set $\Omega_{v} \equiv 0$.) In particular, we have for each $n \in \mathbb{Z}$, a function $\Omega_{n}$ on $\mathbb{C}$ in the variable $z$, namely

$$
\Omega_{n}(z ; \Lambda)=\frac{\sigma(n z ; \Lambda)}{\sigma(z ; \Lambda)^{n^{2}}},
$$

and for each pair $(m, n) \in \mathbb{Z} \times \mathbb{Z}$, a function $\Omega_{m, n}$ on $\mathbb{C} \times \mathbb{C}$ in variables $z$ and $w$ :

$$
\Omega_{m, n}(z, w ; \Lambda)=\frac{\sigma(m z+n w ; \Lambda)}{\sigma(z ; \Lambda)^{m^{2}-m n} \sigma(z+w ; \Lambda)^{m n} \sigma(w ; \Lambda)^{n^{2}-m n}} .
$$

Remark 3.2. Compare the proof of Lemma 4.5 to this definition.

Proposition 3.3. Fix a lattice $\Lambda \in \mathbb{C}$ corresponding to an elliptic curve $E$. The functions $\Omega_{v}$ are elliptic functions in each variable.

Proof. Let $\omega \in \Lambda$. We show the function is elliptic in the first variable. Let $\boldsymbol{v}=\left(v_{1}, \ldots, v_{n}\right) \in \mathbb{Z}^{n}$ and $z=\left(z_{1}, \ldots, z_{n}\right), \boldsymbol{w}=(\omega, 0, \ldots, 0) \in \mathbb{C}^{n}$. Using (15), we calculate

$$
\frac{\Omega_{v}(z+w ; \Lambda)}{\Omega_{v}(z ; \Lambda)}=\frac{\lambda\left(v_{1} \omega\right)}{\lambda(\omega)^{v_{1}^{2}}}=1
$$

where the last equality holds because $\lambda$ is a quadratic form. Thus $\Omega_{v}$ is invariant under adding a period to the variable $z_{1}$. Similarly $\Omega_{v}$ is elliptic in each variable on $(\mathbb{C} / \Lambda)^{n}$.

Proposition 3.4. Fix a lattice $\Lambda \in \mathbb{C}$. Let $\boldsymbol{v} \in \mathbb{Z}^{m}$ and $z \in \mathbb{C}^{n}$. Let $T$ be an $n \times m$ matrix with entries in $\mathbb{Z}$ and transpose $T^{\text {tr }}$. Then

$$
\Omega_{\boldsymbol{v}}\left(T^{t r}(\boldsymbol{z}) ; \Lambda\right)=\frac{\Omega_{T(\boldsymbol{v})}(z ; \Lambda)}{\prod_{i=1}^{n} \Omega_{T\left(\boldsymbol{e}_{i}\right)}(\boldsymbol{z} ; \Lambda)^{2 v_{i}^{2}-\sum_{j=1}^{n} v_{i} v_{j}} \prod_{1 \leq i<j \leq n} \Omega_{T\left(\boldsymbol{e}_{i}+\boldsymbol{e}_{j}\right)}(\boldsymbol{z} ; \Lambda)^{v_{i} v_{j}}} .
$$

Proof. A straightforward calculation using Definition 3.1.

Let $\wp$ and $\zeta$ denote the usual Weierstrass functions. 


\section{Lemma 3.5.}

$$
\begin{array}{r}
\wp(u)-\wp(v)=-\frac{\sigma(u+v) \sigma(u-v)}{\sigma(u)^{2} \sigma(v)^{2}} . \\
\wp(\boldsymbol{v} \cdot \boldsymbol{z})-\wp(\boldsymbol{w} \cdot \boldsymbol{z})=-\frac{\Omega_{\boldsymbol{v}+\boldsymbol{w}}(\boldsymbol{z}) \Omega_{\boldsymbol{v}-\boldsymbol{w}}(\boldsymbol{z})}{\Omega_{\boldsymbol{v}}(\boldsymbol{z})^{2} \Omega_{\boldsymbol{w}}(\boldsymbol{z})^{2}} .
\end{array}
$$

Proof. Part (a) is well-known; see [Chandrasekharan 1985], for example. Part (b) follows by direct calculation using Definition 3.1.

\section{Lemma 3.6.}

(a) $\zeta(x+a)-\zeta(a)-\zeta(x+b)+\zeta(b)=\frac{\sigma(x+a+b) \sigma(x) \sigma(a-b)}{\sigma(x+a) \sigma(x+b) \sigma(a) \sigma(b)}$.

(b) $\zeta(x+a+b)-\zeta(x+a)-\zeta(x+b)+\zeta(x)=\frac{\sigma(2 x+a+b) \sigma(a) \sigma(b)}{\sigma(x+a+b) \sigma(x+a) \sigma(x+b) \sigma(x)}$.

Proof. (a) Denote by $f$ and $g$ the two sides of the equation to be proved. Considered as functions of any one of $x, a$ or $b$, these are elliptic functions. Suppose that $a, b \notin \Lambda$. Consider $f$ and $g$ as functions of $x$. The set of poles of $f$ or $g$ is $\{-a,-b\}$. The zeroes of $g$ (the right-hand side) are at $-a-b$ and 0 . These are also zeroes of $f$, since $\zeta$ is an odd function. Hence $f=c g$ for some $c$ not depending on $x$. Now define instead

$$
\begin{aligned}
& F=(\zeta(x+a)-\zeta(a)-\zeta(x+b)+\zeta(b)) \sigma(x+a) \sigma(x+b), \\
& G=\sigma(x+a+b) \sigma(x) .
\end{aligned}
$$

We have $F=c^{\prime} G$ for some constant $c^{\prime}$ independent of $x$. Taking derivatives and evaluating at $x=0$, we have

$$
(\wp(b)-\wp(a)) \sigma(a) \sigma(b)=c^{\prime} \sigma(a+b) \sigma^{\prime}(0)
$$

We have $\sigma^{\prime}(0)=1$. By Lemma 3.5, we then have

$$
c^{\prime}=-\frac{\sigma(a-b)}{\sigma(a) \sigma(b)}
$$

which concludes the proof of (a). Part (b) is obtained by a change of variables $x \leftarrow a, a \leftarrow x+b, b \leftarrow x$.

\section{Forming the elliptic net.}

Theorem 3.7. Fix a lattice $\Lambda \in \mathbb{C}$ corresponding to an elliptic curve E. Fix $z_{1}, \ldots, z_{n} \in \mathbb{C}$. Then the function $W: \mathbb{Z}^{n} \rightarrow \mathbb{C}$ defined by

$$
W(\boldsymbol{v})=\Omega_{\boldsymbol{v}}\left(z_{1}, \ldots, z_{n} ; \Lambda\right)
$$

is an elliptic net. 
Proof. For notational simplicity, we drop the arguments $z_{i}, \Lambda$ on $\Omega_{v}$ and also write $\sigma(\boldsymbol{v}), \wp(\boldsymbol{v})$ and $\zeta(\boldsymbol{v})$ for $\sigma\left(v_{1} z_{1}+\cdots+v_{n} z_{n}\right), \wp\left(v_{1} z_{1}+\cdots+v_{n} z_{n}\right)$ and $\zeta\left(v_{1} z_{1}+\cdots+v_{n} z_{n}\right)$. We observe that $\boldsymbol{v}=\mathbf{0}$ if and only if $\Omega_{v} \equiv 0$.

We intend to show that (3) holds for $W$ in $\boldsymbol{p}, \boldsymbol{q}, \boldsymbol{r}$ and $\boldsymbol{s}$. If any one of $\boldsymbol{p}$, $\boldsymbol{q}$ or $\boldsymbol{r}$ are zero, then (3) holds trivially (note that $\sigma$ is an odd function, so that $\Omega_{-v}=-\Omega_{v}$ ). Hence we may assume that none of $\Omega_{p}, \Omega_{q}$, or $\Omega_{r}$ is identically zero. For any quadratic form $f$ defined on $\mathbb{Z}^{n}$, we have the following relation for all $\boldsymbol{p}, \boldsymbol{q}, \boldsymbol{s} \in \mathbb{Z}^{n}$ :

$$
f(\boldsymbol{p}+\boldsymbol{q}+\boldsymbol{s})+f(\boldsymbol{p}-\boldsymbol{q})+f(\boldsymbol{s})-f(\boldsymbol{p}+\boldsymbol{s})-f(\boldsymbol{p})-f(\boldsymbol{q}+\boldsymbol{s})-f(\boldsymbol{q})=0 .
$$

First we address the case that $\boldsymbol{s}=\mathbf{0}$. By (16) and Lemma 3.5,

$$
\frac{\Omega_{\boldsymbol{p}+\boldsymbol{q}} \Omega_{\boldsymbol{p}-\boldsymbol{q}}}{\Omega_{\boldsymbol{p}}^{2} \Omega_{\boldsymbol{q}}^{2}}=\frac{\sigma(\boldsymbol{p}+\boldsymbol{q}) \sigma(\boldsymbol{p}-\boldsymbol{q})}{\sigma(\boldsymbol{p})^{2} \sigma(\boldsymbol{q})^{2}}=\wp(\boldsymbol{q})-\wp(\boldsymbol{p}) .
$$

Therefore, we have

$$
\frac{\Omega_{p+q} \Omega_{p-q}}{\Omega_{p}^{2} \Omega_{q}^{2}}+\frac{\Omega_{q+r} \Omega_{q-r}}{\Omega_{q}^{2} \Omega_{r}^{2}}+\frac{\Omega_{r+p} \Omega_{r-p}}{\Omega_{r}^{2} \Omega_{p}^{2}}=0,
$$

which gives the relation (3) for $\boldsymbol{s}=\mathbf{0}$, that is,

$$
\Omega_{p+q} \Omega_{p-q} \Omega_{r}^{2}+\Omega_{q+r} \Omega_{q-r} \Omega_{p}^{2}+\Omega_{r+p} \Omega_{r-p} \Omega_{q}^{2}=0 .
$$

Now suppose that $\boldsymbol{s} \neq \mathbf{0}$ and so $\Omega_{\boldsymbol{s}} \not \equiv 0$. By (16) and Lemma 3.6,

$$
\begin{aligned}
\frac{\Omega_{\boldsymbol{p}+\boldsymbol{q}+s} \Omega_{\boldsymbol{p}-\boldsymbol{q}} \Omega_{s}}{\Omega_{\boldsymbol{p}+s} \Omega_{p} \Omega_{\boldsymbol{q}+s} \Omega_{\boldsymbol{q}}} & =\frac{\sigma(\boldsymbol{p}+\boldsymbol{q}+\boldsymbol{s}) \sigma(\boldsymbol{p}-\boldsymbol{q}) \sigma(\boldsymbol{s})}{\sigma(\boldsymbol{p}+\boldsymbol{s}) \sigma(\boldsymbol{p}) \sigma(\boldsymbol{q}+\boldsymbol{s}) \sigma(\boldsymbol{q})} \\
& =\zeta(\boldsymbol{p}+\boldsymbol{s})-\zeta(\boldsymbol{p})-\zeta(\boldsymbol{q}+\boldsymbol{s})+\zeta(\boldsymbol{q})
\end{aligned}
$$

Therefore, we have

$$
\frac{\Omega_{p+q+s} \Omega_{p-q} \Omega_{s}}{\Omega_{p+s} \Omega_{p} \Omega_{q+s} \Omega_{q}}+\frac{\Omega_{q+r+s} \Omega_{q-r} \Omega_{s}}{\Omega_{q+s} \Omega_{q} \Omega_{r+s} \Omega_{r}}+\frac{\Omega_{r+p+s} \Omega_{r-p} \Omega_{s}}{\Omega_{r+s} \Omega_{r} \Omega_{p+s} \Omega_{p}}=0,
$$

or, more simply,

$$
\Omega_{p+q+s} \Omega_{p-q} \Omega_{r+s} \Omega_{r}+\Omega_{q+r+s} \Omega_{q-r} \Omega_{p+s} \Omega_{p}+\Omega_{r+p+s} \Omega_{r-p} \Omega_{q+s} \Omega_{q}=0,
$$

which is what was required to prove.

The identity (3) for $\Omega_{v}$ is similar to several identities known in complex function theory [Gasper and Rahman 2004; Wenchang et al. 1996]. 
Explicit rational functions. Elliptic functions for a lattice $\Lambda$ of $\mathbb{C}$ give rational functions on the associated elliptic curve (via complex uniformization). If we give a Weierstrass model for the same elliptic curve, we can give explicit expressions for the rational functions as elements of the usual field of rational functions associated to the model. In the following proposition, we do this for $\Omega_{v}$ for some small $v \in \mathbb{Z}^{n}$, for $n=1,2,3$.

Proposition 3.8. Consider an elliptic curve $E$, and a Weierstrass model for $E$ given by

$$
y^{2}+a_{1} x y+a_{3} y-x^{3}-a_{2} x^{2}-a_{4} x-a_{6}=0 .
$$

As usual, let

$$
\begin{gathered}
b_{2}=a_{1}^{2}+4 a_{2}, \quad b_{4}=2 a_{4}+a_{1} a_{3}, \quad b_{6}=a_{3}^{2}+4 a_{6}, \\
b_{8}=a_{1}^{2} a_{6}+4 a_{2} a_{6}-a_{1} a_{3} a_{4}+a_{2} a_{3}^{2}-a_{4}^{2} .
\end{gathered}
$$

To $E$ we can also associate a complex uniformization and elliptic functions $\Omega_{v}$ as above. As rational functions on $E$, we have the following equalities.

For $n=1$ :

$$
\begin{aligned}
& \Omega_{1}=1, \quad \Omega_{2}=2 y+a_{1} x+a_{3}, \\
& \Omega_{3}=3 x^{4}+b_{2} x^{3}+3 b_{4} x^{2}+3 b_{6} x+b_{8}, c r \Omega_{4}=\left(2 y+a_{1} x+a_{3}\right) \\
& \quad\left(2 x^{6}+b_{2} x^{5}+5 b_{4} x^{4}+10 b_{6} x^{3}+10 b_{8} x^{2}+\left(b_{2} b_{8}-b_{4} b_{6}\right) x+b_{4} b_{8}-b_{6}^{2}\right) .
\end{aligned}
$$

For $n=2$ :

$$
\begin{aligned}
& \Omega_{(1,0)}=\Omega_{(0,1)}=\Omega_{(1,1)}=1, \\
& \Omega_{(1,-1)}=x_{2}-x_{1}, \quad \Omega_{(-1,1)}=x_{1}-x_{2}, \\
& \Omega_{(2,1)}=2 x_{1}+x_{2}-\left(\frac{y_{2}-y_{1}}{x_{2}-x_{1}}\right)^{2}-a_{1}\left(\frac{y_{2}-y_{1}}{x_{2}-x_{1}}\right)+a_{2}, \\
& \Omega_{(1,2)}=x_{1}+2 x_{2}-\left(\frac{y_{2}-y_{1}}{x_{2}-x_{1}}\right)^{2}-a_{1}\left(\frac{y_{2}-y_{1}}{x_{2}-x_{1}}\right)+a_{2} .
\end{aligned}
$$

For $n=3$ :

$$
\begin{aligned}
& \Omega_{(1,0,0)}=\Omega_{(0,1,0)}=\Omega_{(0,0,1)}=\Omega_{(1,1,0)}=\Omega_{(0,1,1)}=\Omega_{(1,0,1)}=1, \\
& \Omega_{(1,-1,0)}=x_{2}-x_{1}, \quad \Omega_{(0,1,-1)}=x_{3}-x_{2}, \quad \Omega_{(-1,0,1)}=x_{1}-x_{3}, \\
& \Omega_{(-1,1,0)}=x_{1}-x_{2}, \quad \Omega_{(0,-1,1)}=x_{2}-x_{3}, \quad \Omega_{(1,0,-1)}=x_{3}-x_{1}, \\
& \Omega_{(1,1,1)}=\frac{y_{1}\left(x_{2}-x_{3}\right)+y_{2}\left(x_{3}-x_{1}\right)+y_{3}\left(x_{1}-x_{2}\right)}{\left(x_{1}-x_{2}\right)\left(x_{1}-x_{3}\right)\left(x_{2}-x_{3}\right)},
\end{aligned}
$$




$$
\begin{aligned}
& \Omega_{(-1,1,1)}=\frac{y_{1}\left(x_{2}-x_{3}\right)-y_{2}\left(x_{3}-x_{1}\right)-y_{3}\left(x_{1}-x_{2}\right)}{\left(x_{2}-x_{3}\right)}+a_{1} x_{1}+a_{3}, \\
& \Omega_{(1,-1,1)}=\frac{-y_{1}\left(x_{2}-x_{3}\right)+y_{2}\left(x_{3}-x_{1}\right)-y_{3}\left(x_{1}-x_{2}\right)}{\left(x_{3}-x_{1}\right)}+a_{1} x_{2}+a_{3}, \\
& \Omega_{(1,1,-1)}=\frac{-y_{1}\left(x_{2}-x_{3}\right)-y_{2}\left(x_{3}-x_{1}\right)+y_{3}\left(x_{1}-x_{2}\right)}{\left(x_{1}-x_{2}\right)}+a_{1} x_{3}+a_{3} .
\end{aligned}
$$

Proof. The division polynomial formulae (the $n=1$ case) are well-known; see [Chandrasekharan 1985], [Frey and Lange 2006, p. 80], or [Silverman 2009, Exercise 3.7]. The formulae for $n=2$ and the related first three lines of formulae for $n=3$ are immediate consequences of Lemma 3.5 and the addition law for elliptic curves [Silverman 2009, Algorithm 2.3]. Only the cases where $n=3, v_{i} \neq 0$ for all $i=1,2,3$ are not immediate: these formulae are a result of the proof of Lemma 2.6. Note that using Remark 2.7 results in the same formulae.

\section{Net polynomials over arbitrary fields}

In the last section, we defined elliptic functions $\Omega_{v}$ in the case of $\mathbb{C} / \Lambda$. In this section we wish to define the same rational functions for any elliptic curve over any field, calling them $\Psi_{v}$, the net polynomials. We will start from the results of the last section.

Defining net polynomials. Let $R=\mathbb{Q}\left[\alpha_{1}, \alpha_{2}, \alpha_{3}, \alpha_{4}, \alpha_{6}\right]$ be a polynomial ring over $\mathbb{Q}$ in the variables $\alpha_{i}$. Define $f(x, y) \in R[x, y]$ by

$$
f(x, y)=y^{2}+\alpha_{1} x y+\alpha_{3} y-x^{3}-\alpha_{2} x^{2}-\alpha_{4} x-\alpha_{6} .
$$

Consider the affine scheme $\mathscr{E}: f(x, y)=0$ over $R$. Let $\boldsymbol{a}=\left(a_{i}\right) \in \mathbb{C}^{5}$. The association $\left(\alpha_{i}\right) \mapsto\left(a_{i}\right)$ gives a map $\phi_{\boldsymbol{a}}: R \rightarrow \mathbb{C}$. Consider the affine variety over $\mathbb{C}$ given by

$$
C_{\boldsymbol{a}}: y^{2}+a_{1} x y+a_{3} y=x^{3}+a_{2} x^{2}+a_{4} x+a_{6} .
$$

Then $\phi_{a}$ gives rise to a Cartesian diagram



where $\mathscr{E}^{n}=\mathscr{E} \times \operatorname{Spec} R \cdots \times \operatorname{Spec} R \mathscr{E}$ is the $n$-fold fibre product of $\mathscr{E}$ with itself over $R$.

The rational functions $\Omega_{v} \in \mathscr{K}\left(C_{a}^{n}\right)$ have rational expressions in $x, y$ and the $a_{i}$ (in terms of the Weierstrass model, as in for example Proposition 3.8). These expressions have rational coefficients by construction and the general theory of 
sigma functions (the divisors are Galois invariant). So these same expressions (with $a_{i}$ replaced with $\alpha_{i}$ ) give rational functions $\Psi_{v} \in \mathscr{K}\left(\mathscr{E}^{n}\right)$.

Theorem 4.1. Let $n \geq 1$. Denote by $\mathscr{K}\left(\mathscr{E}^{n}\right)$ the field of rational functions on $\mathscr{E}^{n}$. There exists a unique system of functions $\Psi_{v} \in \mathscr{K}\left(\mathscr{E}^{n}\right)$ depending on $\boldsymbol{v} \in \mathbb{Z}^{n}$ such that

(a) the map

$$
W: \mathbb{Z}^{n} \rightarrow \mathscr{K}\left(\mathscr{E}^{n}\right), \quad \boldsymbol{v} \mapsto \Psi_{v}
$$

is an elliptic net, and

(b) whenever $C_{a}$ is elliptic, the restriction of $\Psi_{v}$ to a fibre $C_{a}^{n}$ is the rational function $\Omega_{v}$.on $C_{a}^{n}$.

Proof. The union of the $C_{\boldsymbol{a}}^{n}$ for which $C_{\boldsymbol{a}}$ is an elliptic curve is Zariski dense, and so the $\Psi_{v}$ are determined uniquely by their restrictions to these fibres.

We call these $\Psi_{v}$ the net polynomials; we will discuss shortly the "polynomial" ring $\mathscr{R}_{n}$ in which they live.

We transfer some useful properties of the $\Omega_{v}$ to properties of the $\Psi_{v}$ on $\mathscr{E}^{n}$. Again, there are unique rational functions $X$ and $Y$ for $\mathscr{E}$ whose restriction to elliptic $C_{\boldsymbol{a}}$ correspond to the Weierstrass functions $\wp$ and $\frac{1}{2} \wp^{\prime}$. Each $\boldsymbol{v} \in \mathbb{Z}^{n}$ gives rise to a map $v: \mathscr{E}^{n} \rightarrow \mathscr{E}$ which is the linear combination associated to the vector $\boldsymbol{v}$ (e.g., $(1,1)$ is the usual group law). Define rational functions $X_{v}=X \circ v$ and $Y_{\boldsymbol{v}}=Y \circ \boldsymbol{v}$ on $\mathscr{E}^{n}$.

The next lemma follows immediately from Lemma 3.5.

Lemma 4.2.

$$
\Psi_{v}^{2} \Psi_{w}^{2}\left(X_{v}-X_{w}\right)=-\Psi_{v+w} \Psi_{v-w} .
$$

More generally, there is a map $T: \mathscr{C}^{m} \rightarrow \mathscr{\mathscr { C }}^{n}$ associated to any $T \in M_{n \times m}(\mathbb{Z})$. The next proposition follows from Proposition 3.4.

Proposition 4.3. Let $\boldsymbol{v} \in \mathbb{Z}^{n}$. Let $T$ be any $n \times m$ matrix with entries in $\mathbb{Z}$ and transpose $T^{\text {tr. }}$. Then

$$
\left(\Psi_{\boldsymbol{v}} \circ T\right) \prod_{i=1}^{n} \Psi_{T^{t r}\left(\boldsymbol{e}_{i}\right)}^{2 v_{j}^{2}-\sum_{j=1}^{n} v_{i} v_{j}} \prod_{1 \leq i<j \leq n} \Psi_{T^{t r}\left(\boldsymbol{e}_{i}+\boldsymbol{e}_{j}\right)}^{v_{i} v_{j}}=\Psi_{T^{t r}(\boldsymbol{v})} .
$$

Net polynomials at primes. In this section we determine a little more about the exact nature of the elliptic net $\Psi_{v}$. In particular, we wish to restrict the possible divisor of $\Psi_{v}$, and show that it has zero valuation for certain primes.

Consider the ring $S=\mathbb{Z}\left[\alpha_{1}, \alpha_{2}, \alpha_{3}, \alpha_{4}, \alpha_{6}\right]$. Since $f(x, y)$ is defined over $S$, we may define $\mathscr{E}_{S}: f(x, y)=0$ as a scheme over $\operatorname{Spec} S$ whose fibre over $\operatorname{Spec} R$ is 
$\mathscr{E}$. Then $\mathscr{E}_{S}^{n}=\mathscr{E}_{S} \times \times_{\operatorname{Spec} S} \cdots \times \times_{\operatorname{Spec} S} \mathscr{E}_{S}$ is a scheme over Spec $S$ whose fibre over Spec $R$ is $\mathscr{C}^{n}$. Define

$$
\mathscr{R}_{n}=S\left[x_{i}, y_{i}\right]_{1 \leq i \leq n}\left[\left(x_{i}-x_{j}\right)^{-1}\right]_{1 \leq i<j \leq n} /\left\langle f\left(x_{i}, y_{i}\right)\right\rangle_{1 \leq i \leq n} .
$$

The ring $\mathscr{R}_{n}$ is the affine coordinate ring of the affine piece of $\mathscr{C}_{S}^{n}$ obtained by removing all the diagonals and antidiagonals, in the sense of the elliptic curve group law (in other words, on an elliptic curve fibre, $x_{i}=x_{j}$ if and only if the corresponding points satisfy $P_{i}= \pm P_{j}$ ). There is a natural identification of $\mathscr{R}_{n}$ with a subset of $\mathscr{K}\left(\mathscr{E}^{n}\right)$.

Theorem 4.4. The functions $\Psi_{v}$ are elements of $\mathscr{R}_{n}$. Let $\mathfrak{p}$ be any prime of $\mathscr{R}_{n}$ which is a lift of a prime of $S$. Then $\Psi_{v} \notin \mathfrak{p}$.

The lifted ideal $\mathfrak{p}=\mathfrak{q} \mathscr{R}_{n}$ is prime whenever $\mathfrak{q}$ is a prime of $S$. The proof of the theorem will involve showing for all valuations $v$ associated to such primes $\mathfrak{p}$ that $v\left(\Psi_{v}\right)$ (slightly modified) is a quadratic form with certain vanishing. Then the following lemma will establish that this function is identically zero.

Let $B$ and $C$ be abelian groups written additively. The function $f: B \rightarrow C$ is a quadratic form if for all $x, y, z \in B$,

$$
f(x+y+z)-f(x+y)-f(y+z)-f(x+z)+f(x)+f(y)+f(z)=0 .
$$

If $f$ is a quadratic form, then for all $x, y \in B$,

$$
f(x+y)+f(x-y)-2 f(x)-2 f(y)=0 .
$$

The converse holds if $C$ is 2-torsion free.

Lemma 4.5. Let $M: \mathbb{Z}^{n} \rightarrow \mathbb{Z}$ be a quadratic form. Suppose that $M(v)=0$ for all $\boldsymbol{v}=\boldsymbol{e}_{i}$ and $\boldsymbol{v}=\boldsymbol{e}_{i}+\boldsymbol{e}_{j}$ (i.e., for standard basis vectors and their two-term sums). Then $M(v)=0$ for all $\boldsymbol{v}$.

Proof. It is well-known that any value of a quadratic form can be given in terms of its value at a certain "base" of vectors. In particular,

$$
f\left(\sum_{i=1}^{n} a_{i} \boldsymbol{e}_{i}\right)=\sum_{i=1}^{n}\left(2 a_{i}^{2}-\sum_{j=1}^{n} a_{i} a_{j}\right) f\left(\boldsymbol{e}_{i}\right)+\sum_{1 \leq i<j \leq n} a_{i} a_{j} f\left(\boldsymbol{e}_{i}+\boldsymbol{e}_{j}\right) .
$$

Proof of Theorem 4.4. Each $\Psi_{v} \in \mathscr{K}\left(\mathscr{E}^{n}\right)$ has a corresponding Weil divisor. Suppose a codimension-one subscheme $X$ appears as a summand in this divisor, and let $\widetilde{X}=X \cap C_{\boldsymbol{a}}^{n}$. If $C_{\boldsymbol{a}}$ is elliptic, $\widetilde{X} \neq \varnothing$, and $\widetilde{X} \neq C_{\boldsymbol{a}}^{n}$, then $\widetilde{X}$ is of codimension one in $C_{a}^{n}$ and appears in the divisor of $\Omega_{v}$ to the same order as $X$ appears in the divisor of $\Psi_{v}$. Definition 3.1 determines the divisors of $\Omega_{v}$ and this restricts the possible divisors for $\Psi_{v}$. In particular, it shows that $s \Psi_{v} \in \mathscr{R}_{n}$, where $s \in S$. 
Therefore, taking $v$ to be a valuation of $\mathscr{R}_{n}$ lifted from a valuation of $S$ associated to a prime $\mathfrak{q}$ of $S$, it will suffice to show that $v\left(\Psi_{v}\right)=0$ for all $v \in \mathbb{Z}^{n}$.

Lemma 4.2 implies

$$
X_{v}-X_{w}=-\frac{\Psi_{v+w} \Psi_{v-w}}{\Psi_{v}^{2} \Psi_{w}^{2}} .
$$

We claim that $v\left(X_{\boldsymbol{v}}-X_{\boldsymbol{w}}\right)=0$ whenever $\boldsymbol{v} \neq \pm \boldsymbol{w}, \boldsymbol{v} \neq 0$, and $\boldsymbol{w} \neq 0$.

First suppose $v\left(X_{v}-X_{w}\right)<0$; we show that $v=0$ or $\boldsymbol{w}=0$. Indeed, we know that $v\left(X_{v}\right)<0$ or $v\left(X_{w}\right)<0$. Suppose $v\left(X_{v}\right)<0$. This implies that $\boldsymbol{v}(\boldsymbol{P})=0$ for all $\boldsymbol{P}$ on the nonsingular part of the fibre over $\mathfrak{q}$ of $\mathscr{E}_{S}$. Since $\boldsymbol{P}$ ranges over all possible values (e.g., $\boldsymbol{P}=(P, \mathcal{O}, \ldots, \mathcal{O})$ ), we find that this implies that $\left[v_{i}\right]=[0]$ for all $i$. In turn, this shows that $\boldsymbol{v}=0$. Similarly, if $v\left(X_{\boldsymbol{w}}\right)<0$, then $\boldsymbol{w}=0$.

Next suppose $v\left(X_{v}-X_{\boldsymbol{w}}\right)>0$; we show that $\boldsymbol{v}= \pm \boldsymbol{w}$. Suppose the valuation is positive. Then $\boldsymbol{v}(\boldsymbol{P})= \pm \boldsymbol{w}(\boldsymbol{P})$ for all $\boldsymbol{P}$ on the nonsingular part of the fibre over $\mathfrak{q}$ of $\mathscr{E}_{S}$. Since $\boldsymbol{P}$ ranges over all possible values (e.g., $\boldsymbol{P}=(P, \mathcal{O}, \ldots, \mathcal{O})$ or $\boldsymbol{P}=$ $(P, P, \mathcal{O}, \ldots, \mathcal{O}))$, we find that this implies, in particular, that for all $0 \leq i \leq j \leq n$, we have $\left[v_{i} \pm w_{i}\right]=[0]$ and $\left[v_{i}+v_{j} \pm\left(w_{i}+w_{j}\right)\right]=[0]$ on $\mathscr{E}_{S}$. In turn, this gives $v_{i}= \pm w_{i}$ and $v_{i}+v_{j}= \pm\left(w_{i}+w_{j}\right)$. Together these imply that $\boldsymbol{v}= \pm \boldsymbol{w}$. This demonstrates the claim.

Define a function $M: \mathbb{Z}^{n} \rightarrow \mathbb{Z}$ by

$$
M(\boldsymbol{v})= \begin{cases}v\left(\Psi_{\boldsymbol{v}}\right) & \text { if } \boldsymbol{v} \neq 0 \\ 0 & \text { if } \boldsymbol{v}=0\end{cases}
$$

Note that $M(-\boldsymbol{v})=M(v)$, from which one can deduce that

$$
M(\boldsymbol{v}+\boldsymbol{w})+M(\boldsymbol{v}-\boldsymbol{w})-2 M(\boldsymbol{v})-2 M(\boldsymbol{w})=0
$$

whenever $\boldsymbol{v}=0$ or $\boldsymbol{w}=0$. Our work up until now has shown that (18) holds in all other cases except $\boldsymbol{v}+\boldsymbol{w}=0$ or $\boldsymbol{v}-\boldsymbol{w}=0$. These remaining two cases reduce to the statement that for all $\boldsymbol{u}, M(2 \boldsymbol{u})=4 M(\boldsymbol{u})$. To obtain this, take the sum of the four instances of $(18)$ with $(\boldsymbol{v}, \boldsymbol{w})$ respectively taking the values $(4 \boldsymbol{u}, \boldsymbol{u}),(3 \boldsymbol{u}, \boldsymbol{u})$, $(3 \boldsymbol{u}, \boldsymbol{u})$ and $(2 \boldsymbol{u}, \boldsymbol{u})$, and then subtract the instance of (18) with $(\boldsymbol{v}, \boldsymbol{w})=(3 \boldsymbol{u}, 2 \boldsymbol{u})$.

We have shown that (18) holds for all $v$ and $\boldsymbol{w}$, and that therefore $M: \mathbb{Z}^{n} \rightarrow \mathbb{Z}$ is a quadratic form (since $\mathbb{Z}$ is 2-torsion free). The other assumptions of Lemma 4.5 are verified by Proposition 3.8. Therefore, $M$ is identically zero, which is what was required to prove.

Summary. Let $n \geq 1$. For any elliptic curve or scheme $C$, let $O$ denote the identity, $[m]: C \rightarrow C$ denote multiplication by $m, p_{i}: C^{n} \rightarrow C$ denote projection onto the $i$-th component, and $s: C^{n} \rightarrow C$ denote sum of all components. For $v \in \mathbb{Z}^{n}$, define 
the expression

$$
\begin{aligned}
D_{C, v}=\left(\left[v_{1}\right] \times \cdots \times\left[v_{n}\right]\right)^{*} s^{*}(\mathbb{O})-\sum_{1 \leq k<j \leq n} v_{k} v_{j}\left(p_{k}^{*} \times p_{j}^{*}\right) s^{*}(\mathbb{O}) & \\
& -\sum_{k=1}^{n}\left(2 v_{k}^{2}-\sum_{j=1}^{n} v_{k} v_{j}\right) p_{k}^{*}(\mathbb{O}),
\end{aligned}
$$

which is a divisor on the $n$-fold product $C^{n}$. Over the complex numbers, the functions $\Omega_{v}$ have these divisors and satisfy the elliptic net recurrence (3) (see Section 3).

We now collect the results of the previous sections in one statement.

Theorem 4.6. Let $n \geq 1$. There exists a unique collection of rational functions $\Psi_{v} \in \mathscr{K}\left(\mathscr{C}_{S}^{n}\right)$ for each $\boldsymbol{v} \in \mathbb{Z}^{n}$ satisfying these conditions:

(a) The map $\boldsymbol{v} \mapsto \Psi_{v}$ gives an elliptic net $W: \mathbb{Z}^{n} \rightarrow \mathscr{R}_{n}$.

(b) $\Psi_{v}=1$ whenever $\boldsymbol{v}=\boldsymbol{e}_{i}$ for some $1 \leq i \leq n$ or $\boldsymbol{v}=\boldsymbol{e}_{i}+\boldsymbol{e}_{j}$ for some $1 \leq i<j \leq n$.

(c) $\operatorname{Div}\left(\Psi_{v}\right)=D_{\mathscr{E}_{S}, v}$.

Proof. Part (a) follows from Theorems 4.1 and 4.4. Part (b) follows from Proposition 3.8 and Theorem 4.1. Part (c) follows from Theorem 4.4.

\section{Elliptic nets from elliptic curves}

In light of Theorem 4.6, it is now natural to define an elliptic net associated to any cubic Weierstrass curve over any field.

Definition 5.1. Let $K$ be any field. Let $a_{1}, a_{2}, a_{3}, a_{4}, a_{6} \in K$. To this we associate a map

$$
S=\mathbb{Z}\left[\alpha_{1}, \alpha_{2}, \alpha_{3}, \alpha_{4}, \alpha_{6}\right] \rightarrow K, \quad \alpha_{i} \mapsto a_{i}
$$

Let

$$
f(x, y)=y^{2}+a_{1} x y+a_{3} y-x^{3}-a_{2} x^{2}-a_{4} x-a_{6}
$$

and let $C$ be a curve defined by $f(x, y)=0$. Then we have a Cartesian diagram



under which we may pullback $\Psi_{v}$ to obtain $\phi_{v} \in \mathscr{K}\left(C^{n}\right)$ (this is possible since the fibre on the right is not contained in the support of the divisor of $\Psi_{v}$, by Theorem 4.6). 
The nonsingular points of $C$ defined over $K$, denoted $C_{\mathrm{ns}}(K)$, form a group. We call a set of points $\left\{P_{1}, \ldots, P_{n}\right\}$ on the nonsingular part $C_{\mathrm{ns}}$ of a cubic curve appropriate if

(a) $P_{i} \neq 0$ for all $i$,

(b) [2] $P_{i} \neq 0$ for all $i$,

(c) $P_{i} \neq \pm P_{j}$ for any $i \neq j$,

and

(d) [3] $P_{1} \neq 0$ whenever $n=1$.

If we have an appropriate $n$-tuple of points $\boldsymbol{P} \in C_{\mathrm{ns}}(K)^{n}$, we may define a map

$$
W_{C, \boldsymbol{P}}: \mathbb{Z}^{n} \rightarrow K
$$

by setting $W_{C, \boldsymbol{P}}(\boldsymbol{v})=\phi_{\boldsymbol{v}}(\boldsymbol{P})$. By Theorem 4.6, this will be an elliptic net. This will be called the elliptic net associated to $C$ and $\boldsymbol{P}$.

We have the following additional corollary to Theorem 4.6.

Corollary 5.2. For an elliptic net $W_{C, P}: \mathbb{Z}^{n} \rightarrow K$ associated to a curve $C$ and nonsingular points $\boldsymbol{P}$, we have $W(\boldsymbol{v})=0$ if and only if $\boldsymbol{v}(\boldsymbol{P})=0$ on $C_{\mathrm{ns}}$.

Proof. This follows from the statement that $\Omega_{v}(v \cdot z)=0$ if and only if $v \cdot z \in \Lambda$ (see Section 3).

Example 5.3. In (4) (page 201) we displayed an example elliptic net $W_{E,(P, Q)}$ associated to the elliptic curve and points

$$
E: y^{2}+y=x^{3}+x^{2}-2 x, \quad P=(0,0), \quad Q=(1,0)
$$

Some of the smaller terms of this net can be calculated using Proposition 3.8; for example,

$$
\begin{gathered}
W(0,0)=0, \quad W(1,0)=W(0,1)=W(1,1)=1, \\
W(2,0)=2 y_{1}+a_{1} x_{1}+a_{3}=1, \quad W(0,2)=2 y_{2}+a_{1} x_{2}+a_{3}=1, \\
W(1,-1)=x_{2}-x_{1}=1, \\
W(2,1)=2 x_{1}+x_{2}-\left(\frac{y_{2}-y_{1}}{x_{2}-x_{1}}\right)^{2}-a_{1}\left(\frac{y_{2}-y_{1}}{x_{2}-x_{1}}\right)+a_{2}=2, \\
W(2,-1)=\left(y_{1}+y_{2}\right)^{2}-\left(2 x_{1}+x_{2}\right)\left(x_{1}-x_{2}\right)^{2}=-1 .
\end{gathered}
$$

More terms can be calculated using the recurrence relation (3). Since $P$ and $Q$ are independent nontorsion points, there are no zeroes in the array except the zero located at the origin $(W(0,0)=0)$. The row through the term 0 is the elliptic divisibility sequence associated to $E$ and $P$, which begins

$1,1,-3,11,38,249,-2357,8767,496035,-3769372,-299154043$,

$$
-12064147359,632926474117,-65604679199921, \ldots
$$

The column through 0 is the elliptic divisibility sequence associated to $Q$. 


\section{Elliptic curves from elliptic nets}

We are now in a position to use the results of Section 2 to determine exactly which elliptic curves (or more generally cubic Weierstrass curves) give rise to any given elliptic net.

\section{Scale equivalence and normalization.}

Proposition 6.1. Let $W: A \rightarrow K$ be an elliptic net. Let $f: A \rightarrow K^{*}$ be a quadratic form. Define $W^{f}: A \rightarrow K$ by

$$
W^{f}(\boldsymbol{v})=f(\boldsymbol{v}) W(\boldsymbol{v}) .
$$

Then $W^{f}$ is an elliptic net.

Proof. Let $p, q, r, s \in A$. We use multiplicative notation in $K^{*}$, so that $f$ satisfies

$$
f(p+q+s) f(p) f(q) f(s) f(p+q)^{-1} f(q+s)^{-1} f(p+s)^{-1}=1 .
$$

The parallelogram law for quadratic forms (written multiplicatively) states that

$$
f(p-q) f(p+q)=f(p)^{2} f(q)^{2} .
$$

Multiplying $f(r) f(r+s)$ and equations (19) and (20) together, we obtain $f(p+q+s) f(p-q) f(r+s) f(r)=f(q+s) f(p+s) f(r+s) f(p) f(q) f(r) f(s)^{-1}$, which is symmetric in $p, q$, and $r$, so

$$
\begin{aligned}
f(p+q+s) f(p-q) f(r+s) f(r) & =f(q+r+s) f(q-r) f(p+s) f(p) \\
& =f(r+p+s) f(r-p) f(q+s) f(q),
\end{aligned}
$$

which shows that the recurrence (3) holds for $W^{f}$ if it does for $W$.

If two elliptic nets are related in the manner of $W$ and $W^{f}$ for some quadratic form $f$, then we call them scale equivalent. This is clearly an equivalence relation.

Let $W: \mathbb{Z}^{n} \rightarrow K$ be an elliptic net. We say that $W$ is normalized if $W\left(\boldsymbol{e}_{i}\right)=1$ for all $1 \leq i \leq n$ and $W\left(\boldsymbol{e}_{i}+\boldsymbol{e}_{j}\right)=1$ for all $1 \leq i<j \leq n$. An elliptic net arising from a curve and points is normalized. It should be stressed that the concept of normalized is only defined for elliptic nets with a preferred basis.

If any term of the form $W\left(\boldsymbol{e}_{i}\right), W\left(2 \boldsymbol{e}_{i}\right), W\left(\boldsymbol{e}_{i}+\boldsymbol{e}_{j}\right)$, or $W\left(\boldsymbol{e}_{i}-\boldsymbol{e}_{j}\right)$ is zero (where $i \neq j)$, or if $n=1$ and any term of the form $W\left(3 \boldsymbol{e}_{1}\right)$ is zero, then we say that $W$ is degenerate. Compare the definition of degenerate to the definition of appropriate in Section 5.

Proposition 6.2. If $W: \mathbb{Z}^{n} \rightarrow K$ is a nondegenerate elliptic net, there is exactly one scaling $W^{f}$ which is normalized. 
Proof. Define

$$
\begin{aligned}
A_{i i} & =W\left(\boldsymbol{e}_{i}\right)^{-1}, \quad \text { for } 1 \leq i \leq n, \\
A_{i j} & =\frac{W\left(\boldsymbol{e}_{i}\right) W\left(\boldsymbol{e}_{j}\right)}{W\left(\boldsymbol{e}_{i}+\boldsymbol{e}_{j}\right)}, \quad \text { for } 1 \leq i<j \leq n, \\
f(\boldsymbol{v}) & =\prod_{1 \leq i \leq j \leq n} A_{i j}^{v_{i} v_{j}} .
\end{aligned}
$$

Then $W^{f}$ is normalized. Uniqueness follows from the elementary properties of quadratic forms (as in the proof of Lemma 4.5).

The proof demonstrates that scale equivalence has $\left(\begin{array}{c}n+1 \\ 2\end{array}\right)$ degrees of freedom. If $W: \mathbb{Z}^{n} \rightarrow K$ is an elliptic net, then its normalization $\widetilde{W}$ is defined to be the unique normalized elliptic net which is a scaling of W. A coordinate sublattice of $\mathbb{Z}^{n}$ is a sublattice of the form

$$
\left\{\boldsymbol{v} \in \mathbb{Z}^{n}: v_{i}=0 \text { for } i \notin I\right\}
$$

for some proper nonempty subset $I \subset\{1,2, \ldots, n\}$. The rank of the sublattice is the cardinality of $I$.

Curves from nets of ranks 1 and 2. Define a change of variables of a cubic curve in Weierstrass form to be unihomothetic if it is of the form

$$
x^{\prime}=x+r, \quad y^{\prime}=y+s x+t,
$$

for some $r, s$ and $t$.

The rank-one result in the following form is due to Christine Swart.

Proposition 6.3 [Swart 2003, Theorem 4.5.3]. Let $W: \mathbb{Z} \rightarrow K$ be a normalized nondegenerate elliptic net. Then the family of curve-point pairs $(C, P)$ such that $W=W_{C, P}$ is three dimensional. These are the curve and nonsingular point

$$
C: y^{2}+a_{1} x y+a_{3} y=x^{3}+a_{2} x^{2}+a_{4} x+a_{6}, \quad P=(0,0),
$$

where

$$
\begin{aligned}
& a_{1}=\frac{W(4)+W(2)^{5}-2 W(2) W(3)}{W(2)^{2} W(3)}, \\
& a_{2}=\frac{W(2) W(3)^{2}+\left(W(4)+W(2)^{5}\right)-W(2) W(3)}{W(2)^{3} W(3)}, \\
& a_{3}=W(2), \quad a_{4}=1,
\end{aligned}
$$

or any image of these under a unihomothetic change of coordinates. 
Proof. A normalized rank 1 nondegenerate elliptic net has $W(2) \neq 0$ and $W(3) \neq 0$. Any singular point $P=(x, y)$ on a cubic Weierstrass curve has vanishing partial derivatives, which implies that $\Psi_{2}(P)=2 y+a_{1} x+a_{3}=0$ (see Proposition 3.8). Therefore, if any curve and singular point gives rise to $W$, then $W(2)=0$, in contradiction to nondegeneracy. The division polynomials $\Psi_{1}, \Psi_{2}, \Psi_{3}$ and $\Psi_{4}$ are invariant under a change of coordinates of the form (21). Then, it is a simple calculation to check that $W_{C, P}$ agrees with $W$ at the first four terms; hence $W_{C, P}=W$ by Theorem 2.2. Conversely, suppose $W=W_{C^{\prime}, P^{\prime}}$. After applying a transformation of the form (21) taking $P^{\prime}$ to $(0,0)$ and taking $a_{4}$ to 1 , substitution of the division polynomials into the equations above verifies that $a_{i}^{\prime}=a_{i}$ for all $i$.

Proposition 6.4. Let $W: \mathbb{Z}^{2} \rightarrow K$ be a normalized nondegenerate elliptic net. Then the family of 3-tuples $\left(C, P_{1}, P_{2}\right)$ such that $W=W_{C, P_{1}, P_{2}}$ is three dimensional. These are the curve and nonsingular points

$$
\begin{gathered}
C: y^{2}+a_{1} x y+a_{3} y=x^{3}+a_{2} x^{2}+a_{4} x+a_{6}, \\
P_{1}=(0,0), \quad P_{2}=(W(1,2)-W(2,1), 0),
\end{gathered}
$$

with

$$
\begin{gathered}
a_{1}=\frac{W(2,0)-W(0,2)}{W(2,1)-W(1,2)}, \quad a_{2}=2 W(2,1)-W(1,2), \quad a_{3}=W(2,0), \\
a_{4}=(W(2,1)-W(1,2)) W(2,1), \quad a_{6}=0,
\end{gathered}
$$

or any image of these under a unihomothetic change of coordinates.

Proof. In a normalized nondegenerate elliptic net,

$$
W(2,1)-W(1,2)=W(1,-1) \neq 0, \quad W(2,0) \neq 0, \quad W(0,2) \neq 0
$$

(see Theorem 2.5). Thus (as in the previous theorem) if a curve and points give rise to $W$, then the points are nonsingular. The formulae for $W(2,0), W(0,2)$, $W(2,1)$ and $W(1,2)$ are invariant under a change of coordinates of the form $(21)$. The net $W_{C, P_{1}, P_{2}}$ agrees with $W$ at the terms $(2,0),(0,2),(2,1)$ and $(1,2)$; hence $W_{C, P_{1}, P_{2}}=W$ by Theorem 2.5. Conversely, suppose $W=W_{C^{\prime}, P_{1}^{\prime}, P_{2}^{\prime}}$. After applying a unihomothetic transformation taking $P_{1}^{\prime}$ to $(0,0)$ and $P_{2}^{\prime}$ to $(W(1,2)-$ $W(2,1), 0)$, substitution of the net polynomials into the equations above verifies that $a_{i}^{\prime}=a_{i}$ for all $i$.

Example 6.5. Plugging terms from the elliptic net of (4) into the formulae in the statement of Proposition 6.4 we recover the corresponding $E, P$, and $Q$.

Remark 6.6. A more symmetric set of equations in the case of characteristic not equal to 2 is as follows:

$$
P_{1}=(v, 0), \quad P_{2}=(-v, 0), \quad 2 v=W(2,1)-W(1,2),
$$




$$
\begin{gathered}
a_{1}=\frac{W(2,0)-W(0,2)}{W(2,1)-W(1,2)}, \quad 2 a_{2}=W(2,1)+W(1,2), \\
2 a_{3}=W(2,0)+W(0,2), \quad 4 a_{4}=-(W(2,1)-W(1,2))^{2}, \\
8 a_{6}=-(W(2,1)-W(1,2))^{2}(W(2,1)+W(1,2)) .
\end{gathered}
$$

\section{Curves from nets in general rank.}

Theorem 6.7. Let $n \geq 1$. Let $W: \mathbb{Z}^{n} \rightarrow K$ be a normalized nondegenerate elliptic net. Then the set of curves $C$ and $\boldsymbol{P} \in C^{n}$ such that $W=W_{C, \boldsymbol{P}}$ forms a three-dimensional family of tuples $(C, \boldsymbol{P})$. Further, none of the points $P \in \boldsymbol{P}$ are singular. In particular, the family consists of one such tuple and all its images under unihomothetic changes of coordinates.

Proof. The proof is by strong induction on $n$, where the inductive statement has two parts:

(I) The theorem holds for $n$.

(II) $W(\boldsymbol{v}) \neq 0$ for some $\boldsymbol{v} \in\{ \pm 1\}^{n}$.

The base case consists of ranks $n=1,2$. Part (I) is by Propositions 6.3 and 6.4; part (II) is by nondegeneracy, which implies $W\left(\boldsymbol{e}_{1}\right) \neq 0$ and $W\left(\boldsymbol{e}_{1}+\boldsymbol{e}_{2}\right) \neq 0$.

Suppose $n \geq 3$ and the inductive statement holds for all $k<n$. Let $W_{1}, \ldots, W_{n}$ be the normalized elliptic subnets of $W$ associated to the rank $n-1$ coordinate sublattices $L_{i}=\left\{\boldsymbol{v}: v_{i}=0\right\}$. These are defined as nets $W_{i}: L_{i} \rightarrow K$ but they can be identified with nets $W_{i}^{\prime}: \mathbb{Z}^{n-1} \rightarrow K$ in the obvious way (by deleting the zero coordinate). They are normalized and nondegenerate (by definition, nondegeneracy at rank $n$ implies nondegeneracy on rank $n-1$ sublattices for $n>2$ ). By part (I) the inductive hypothesis, we have $W_{i}^{\prime}=W_{C_{i}, \boldsymbol{P}_{i}}$ for some curves $C_{i}$ and nonsingular points $\boldsymbol{P}_{i} \in C_{i}^{n-1}$.

We observe a consequence of Proposition 4.3. Suppose $V_{1}: \mathbb{Z}^{m} \rightarrow K$ is an elliptic net of rank $m$ associated to $C$ and $\boldsymbol{P}$. Also suppose

$$
V_{2}:\left\{\boldsymbol{v} \in \mathbb{Z}^{m}: v_{m}=0\right\} \rightarrow K
$$

is the elliptic subnet of $V_{1}$ associated to the coordinate sublattice of rank $m-1$ which consists of vectors with last coordinate zero. Suppose $V_{2}^{\prime}: \mathbb{Z}^{m-1} \rightarrow K$ is naturally identified with $V_{2}$ by simply deleting the last coordinate of the domain. Then $V_{2}^{\prime}$ is associated to $C$ and $\boldsymbol{P}^{\prime}$ where $\boldsymbol{P}^{\prime}$ is simply $\boldsymbol{P}$ with the last coordinate deleted. This statement, appropriately adjusted, holds for any coordinate hyperplane (not just the one with last coordinate zero).

Consider two of the rank $n-1$ subnets, say $W_{i}$ and $W_{j}$. Let $W_{i j}=W_{i} \cap W_{j}$ in $W$. Define $W_{i j}^{\prime}: \mathbb{Z}^{n-2} \rightarrow K$ by the obvious identification. Then, $W_{i j}^{\prime}=W_{C_{i j}, \boldsymbol{P}_{i j}}$ for some curve $C_{i j}$ and $\boldsymbol{P}_{i j} \in C_{i j}^{n-2}$. By the foregoing, $C_{i}=C_{j}=C_{i j}, \boldsymbol{P}_{i j}$ is just $\boldsymbol{P}_{j}$ 
with the $i$-th coordinate deleted, and $\boldsymbol{P}_{i j}$ is just $\boldsymbol{P}_{i}$ with the $(j-1)$-th coordinate deleted.

Considering every such pair, we may define a candidate curve $C$ by $C=C_{i}$ for all $i$ and $\boldsymbol{P} \in C^{n}$ defined as the unique $n$-tuple which results in $\boldsymbol{P}_{i}$ upon deleting the $i$-th coordinate. By the foregoing, this is well-defined. Now we see that $W$ agrees with $W_{C, P}$ on all coordinate sublattices of rank $n-1$. By part (II) of the inductive hypothesis and Theorem 2.8 , we see that $W$ is determined by its sublattices of rank $n-1$. Therefore $W=W_{C, \boldsymbol{P}}$.

To show part (II) of the inductive statement, we observe that if $W(\boldsymbol{v})=0$ for all $\boldsymbol{v} \in\{ \pm 1\}^{n}$, then $\boldsymbol{v}(\boldsymbol{P})=0$ for all such $\boldsymbol{v}$ (by Corollary 5.2). But this is impossible, since it would imply [2] $P_{i}=0$ for $1 \leq i \leq n$, a contradiction to nondegeneracy (again Corollary 5.2).

A change of coordinates of the form (21) for $C$ does not change the elliptic net, as it is determined by its values on its coordinate hyperplanes, where this is true. Further, if two tuples not related by such a change of coordinates generate the same net $W$, then the same would hold for some coordinate hyperplane, a contradiction. This demonstrates part (I) of the inductive statement.

\section{The curve-net theorem}

We set some remaining terminology, and then proceed to the statement and proof of the main theorem.

Homothety and singular elliptic nets. The only changes of coordinates of a Weierstrass equation into another are compositions of unihomothetic changes of coordinates and changes of coordinates of the form $(x, y) \mapsto\left(\lambda^{2} x, \lambda^{3} y\right)$, which we refer to as homotheties (since they correspond to homotheties of the lattice in the complex uniformization).

Proposition 7.1. Consider the rank $n$ elliptic net $W_{C, P}$ associated to

$$
C: y^{2}+a_{1} x y+a_{3} y=x^{3}+a_{2} x^{2}+a_{4} x+a_{6}
$$

defined over $K$ and $\boldsymbol{P} \in C(K)^{n}$. Let $\lambda$ be a nonzero element of $K$. Suppose $\phi_{\lambda}: C \rightarrow C_{\lambda}$ is the isomorphism of curves taking $C$ to

$$
C_{\lambda}: y^{2}+\lambda a_{1} x y+\lambda^{3} a_{3} y=x^{3}+\lambda^{2} a_{2} x^{2}+\lambda^{4} a_{4} x+\lambda^{6} a_{6}
$$

under the change of coordinates $(x, y) \mapsto\left(\lambda^{2} x, \lambda^{3} y\right)$. Then

$$
\widetilde{W}_{C_{\lambda}, \phi_{\lambda}(\boldsymbol{P})}=\lambda \widetilde{W}_{C, \boldsymbol{P}}
$$

In particular, let $\delta_{i j}$ be the Kronecker delta, and define

$$
g(\boldsymbol{v})=-1-\sum_{1 \leq i<j \leq n}(-1)^{\delta_{i j}} v_{i} v_{j}
$$


Then

$$
W_{C_{\lambda}, \phi_{\lambda}(\boldsymbol{P})}=\lambda^{g(\boldsymbol{v})} W_{C, \boldsymbol{P}}
$$

Proof. The first statement is entailed by the second. From the general theory of Weierstrass sigma functions, $\sigma(\lambda z, \lambda \Lambda)=\lambda \sigma(z, \Lambda)$. Thus, by Definition 3.1,

$$
\Omega_{v}(\lambda z ; \lambda \Lambda)=\lambda^{g(v)} \Omega_{v}(z ; \Lambda) .
$$

As in Section 4, this allows us to conclude that the same holds for $\Psi_{v}$, so that

$$
\Psi_{v}\left(\lambda^{2} x, \lambda^{3} y, \lambda^{i} \alpha_{i}\right)=\lambda^{g(v)} \Psi_{v}\left(x, y, \alpha_{i}\right),
$$

from which the result follows.

Definition 7.2. Let $W: \mathbb{Z}^{n} \rightarrow K$ be an elliptic net. With the notation of Proposition 7.1 , we define

$$
W^{\lambda}(\boldsymbol{v}):=\lambda^{g(\boldsymbol{v})} W(\boldsymbol{v}) .
$$

This gives an action of $K$ on elliptic nets $W: \mathbb{Z}^{n} \rightarrow K$ called the homothety action. Two elliptic nets are homothetic if they are in the same orbit of the action of $K$.

The following proposition is immediate.

Proposition 7.3. Let $W: \mathbb{Z}^{n} \rightarrow K$ be an elliptic net. Then for any nonzero $\lambda \in K$, $W^{\lambda}$ is normalized if and only if $W$ is.

Let $W: \mathbb{Z}^{n} \rightarrow K$ be an elliptic net. If the curve $C$ associated to its normalization is a nodal or cuspidal cubic, then $W$ is called singular. If, instead, $C$ is an elliptic curve, then $W$ is called nonsingular. In either case, the discriminant $\Delta$ of $W$ is defined to be the discriminant of the associated Weierstrass equation. Similarly, the $j$-invariant is the $j$-invariant of the associated Weierstrass equation. The discriminant of an elliptic net changes by a factor of $\lambda^{12}$ under homothety, while the $j$-invariant remains unaltered.

The curve-net theorem. We may put a partial ordering on tuples $\left(C, P_{1}, \ldots, P_{n}\right)$ where $C$ is a Weierstrass curve and $P_{i}$ are nonsingular points on the curve. We do this by defining

$$
\left(C, P_{1}, \ldots, P_{n}\right) \leq\left(D, Q_{1}, \ldots, Q_{m}\right)
$$

if and only if $C=D$ and the groups they generate satisfy a containment

$$
\left\langle P_{1}, \ldots, P_{n}\right\rangle \subseteq\left\langle Q_{1}, \ldots, Q_{n}\right\rangle .
$$

The collection of all elliptic nets is partially ordered by the subnet relation. Collecting our work up to this point, we have now shown: 
Theorem 7.4. For each field $K$, there is an explicit isomorphism of partially ordered sets

$$
\begin{gathered}
\left\{\begin{array}{l}
\text { scale equivalence classes of } \\
\text { nondegenerate elliptic nets } \\
W: \mathbb{Z}^{n} \rightarrow K, \text { for some } n
\end{array}\right\} \\
\left\{\begin{array}{l}
\text { tuples }\left(C, P_{1}, \ldots, P_{m}\right) \text { for some } m \text {, where } C \text { is a } \\
\text { cubic curve in Weierstrass form over } K, \text { consid- } \\
\text { ered modulo unihomothetic changes of variables } \\
\text { and such that }\left\{P_{i}\right\} \in C_{\mathrm{ns}}(K)^{m} \text { is appropriate }
\end{array}\right\} .
\end{gathered}
$$

Nonsingular nets correspond to elliptic curves. The action of $K$ (by homothety) on the sets preserves the order and respects the isomorphism. The bijection takes an elliptic net of rank $n$ to a tuple with $n$ points. The elliptic net $W$ associated to a tuple $\left(C, P_{1}, \ldots, P_{n}\right)$ satisfies the property that $W\left(v_{1}, \ldots, v_{n}\right)=0$ if and only if $v_{1} P_{1}+\cdots+v_{n} P_{n}=0$ on the curve $C$.

Proof. In the diagram in the statement of the theorem, call the upper set $\mathcal{N}$ and the lower set $\mathscr{C}$. The first claim is that there is an injective map $\mathcal{N} \rightarrow \mathscr{C}$. Proposition 6.2 shows that each scale equivalence classes in $\mathcal{N}$ contains a unique normalized elliptic net, so we can define the map by Theorem 6.7 (which also guarantees injectivity). Corollary 5.2 shows that the result is an element of $\mathscr{C}$. This shows the first claim.

The second claim is that there exists an inverse map $\mathscr{C} \rightarrow \mathcal{N}$. The map is given by Definition 5.1, which is well-defined as a result of Theorem 4.6. It is required to check that the resulting elliptic net is normalized (Proposition 3.8) and nondegenerate (Corollary 5.2). Theorem 6.7 says that this map is indeed an inverse to the map of the first claim. This gives the second claim and the bijection of sets.

It is clear that the bijection associates an elliptic net of rank $n$ to a tuple with $n$ points, and that it preserves the partial ordering. The action of homothety is preserved by Proposition 7.1. And the final statement of the theorem is a result of Corollary 5.2.

Remark 7.5. The degenerate cases present several difficulties. One is that a degenerate elliptic net may not be determined by the usual initial set of terms as given in Section 2. For example, the sequence given by

$$
W(n)= \begin{cases}0 & \text { if } n \neq k, \\ 1 & \text { if } n=k,\end{cases}
$$

is an elliptic net for any nonzero integer $k$. However, some degenerate sequences can be thought of as arising from singular points on a singular cubic. For example, consider a sequence associated to an elliptic curve $E$ and point $P$ both defined over 
$\mathbb{Q}$ such that $P$ reduces to a singular point modulo some prime $p$. Then the sequence regarded modulo $p$ as living in $\mathbb{F}_{p}$ (which is necessarily a degenerate elliptic net) should be associated to a point on the special fibre of the Néron model. It is likely that Theorem 7.4 can be extended to include these cases (this is future work).

\section{Acknowledgements}

I thank my thesis advisor, Joseph Silverman, for many patient hours. I also thank Rafe Jones, Alf van der Poorten, and Jonathan Wise.

\section{References}

[Ayad 1993] M. Ayad, "Périodicité $(\bmod q)$ des suites elliptiques et points $S$-entiers sur les courbes elliptiques”, Ann. Inst. Fourier (Grenoble) 43:3 (1993), 585-618. MR 94f:11009

[Chandrasekharan 1985] K. Chandrasekharan, Elliptic functions, Grundlehren der Mathematischen Wissenschaften [Fundamental Principles of Mathematical Sciences] 281, Springer, Berlin, 1985. MR 87e:11058 Zbl 0575.33001

[Chudnovsky and Chudnovsky 1986] D. V. Chudnovsky and G. V. Chudnovsky, "Sequences of numbers generated by addition in formal groups and new primality and factorization tests", $A d v$. in Appl. Math. 7:4 (1986), 385-434. MR 88h:11094 Zbl 0614.10004

[Cornelissen and Zahidi 2007] G. Cornelissen and K. Zahidi, "Elliptic divisibility sequences and undecidable problems about rational points", J. Reine Angew. Math. 613 (2007), 1-33. MR2009h: 11196 Zbl 1178.11076

[Eisenträger and Everest 2009] K. Eisenträger and G. Everest, "Descent on elliptic curves and Hilbert's tenth problem", Proc. Amer. Math. Soc. 137:6 (2009), 1951-1959. MR 2009k:11201

[Everest et al. 2003] G. Everest, A. van der Poorten, I. Shparlinski, and T. Ward, Recurrence sequences, Mathematical Surveys and Monographs 104, American Mathematical Society, Providence, RI, 2003. MR 2004c:11015 Zbl 1033.11006

[Everest et al. 2004] G. Everest, V. Miller, and N. Stephens, "Primes generated by elliptic curves", Proc. Amer. Math. Soc. 132:4 (2004), 955-963. MR 2005a:11076 Zbl 1043.11051

[Everest et al. 2006] G. Everest, G. Mclaren, and T. Ward, "Primitive divisors of elliptic divisibility sequences”, J. Number Theory 118:1 (2006), 71-89. MR 2007a:11074 Zbl 0169.15902

[Fomin and Zelevinsky 2002] S. Fomin and A. Zelevinsky, "The Laurent phenomenon", Adv. in Appl. Math. 28:2 (2002), 119-144. MR 2002m:05013 Zbl 1012.05012

[Frey and Lange 2006] G. Frey and T. Lange, "Background on curves and Jacobians", pp. 45-85 in Handbook of elliptic and hyperelliptic curve cryptography, edited by $\mathrm{H}$. Cohen et al., CRC, Boca Raton, FL, 2006. MR 2162720

[Gasper and Rahman 2004] G. Gasper and M. Rahman, Basic hypergeometric series, 2nd ed., Encyclopedia of Mathematics and its Applications 96, Cambridge University Press, Cambridge, 2004. MR 2006d:33028 Zbl 1129.33005

[Hone 2005] A. N. W. Hone, "Elliptic curves and quadratic recurrence sequences", Bull. London Math. Soc. 37:2 (2005), 161-171. MR 2005h:11111 Zbl 1166.11333

[Ingram 2009] P. Ingram, "Multiples of integral points on elliptic curves", J. Number Theory 129:1 (2009), 182-208. MR 2010a:11102 Zbl 05485801 
[Mazur and Tate 1991] B. Mazur and J. Tate, "The p-adic sigma function", Duke Math. J. 62:3 (1991), 663-688. MR 93d:11059 Zbl 0735.14020

[Poonen 2003] B. Poonen, "Hilbert's tenth problem and Mazur's conjecture for large subrings of $\mathbb{Q}$ ", J. Amer. Math. Soc. 16:4 (2003), 981-990. MR 2004f:11145 Zbl 1028.11077

[van der Poorten 2005] A. J. van der Poorten, "Elliptic curves and continued fractions", J. Integer Seq. 8:2 (2005), [article] 05.2.5. MR 2006h:11083

[van der Poorten and Swart 2006] A. J. van der Poorten and C. S. Swart, "Recurrence relations for elliptic sequences: every Somos 4 is a Somos k", Bull. London Math. Soc. 38:4 (2006), 546-554. MR 2007d:11024 Zbl 1169.11013

[Propp 2001] J. Propp, Robbins forum, various messages from January 2, 2001 through March 6, 2001, available at http://faculty.uml.edu/jpropp/about-robbins.txt.

[Shipsey 2001] R. Shipsey, Elliptic divibility sequences, Ph.D. thesis, Goldsmiths, University of London, 2001.

[Silverman 2004] J. H. Silverman, "Common divisors of elliptic divisibility sequences over function fields”, Manuscripta Math. 114:4 (2004), 431-446. MR 2005d:11096 Zbl 1128.11015

[Silverman 2005] J. H. Silverman, " $p$-adic properties of division polynomials and elliptic divisibility sequences”, Math. Ann. 332:2 (2005), 443-474. MR 2006f:11063 Zbl 1066.11024

[Silverman 2009] J. H. Silverman, The arithmetic of elliptic curves, 2nd ed., Graduate Texts in Mathematics 106, Springer, Dordrecht, 2009. MR 2010i:11005 Zbl 1194.11005

[Stange 2007] K. E. Stange, "The Tate pairing via elliptic nets", pp. 329-348 in Pairing-based cryptography: Pairing 2007 (Tokyo, 2007), edited by T. Takagi et al., Lecture Notes in Comput. Sci. 4575, Springer, Berlin, 2007. MR 2009e:11233 Zbl 1151.94570

[Stange 2010] K. E. Stange, Scripts in PARI/GP 2.3.4 and SAGE 4.6.1, 2010, available at http:// math.katestange.net.

[Streng 2008] M. Streng, "Divisibility sequences for elliptic curves with complex multiplication", Algebra Number Theory 2:2 (2008), 183-208. MR 2009e:11110 Zbl 1158.14029

[Swart 2003] C. Swart, Elliptic curves and related sequences, Ph.D. thesis, Royal Holloway and Bedford New College, University of London, 2003.

[Ward 1948] M. Ward, "Memoir on elliptic divisibility sequences", Amer. J. Math. 70 (1948), 31-74. MR 9,332j

[Wenchang et al. 1996] C. Wenchang, S. B. Ekhad, and R. J. Chapman, "Problems and Solutions: Solutions: 10226”, Amer. Math. Monthly 103:2 (1996), 175-177. MR 1542800

Communicated by John H. Coates

Received 2010-04-28 Revised 2010-09-16 Accepted 2010-10-17

stange@math.stanford.edu Department of Mathematics, Stanford University, 450 Serra Mall, Building 380, Stanford, CA, 94305, United States http://math.katestange.net 


\section{Algebra \& Number Theory}

www.jant.org

\section{EDITORS}

MANAGING EDITOR

Bjorn Poonen

Massachusetts Institute of Technology

Cambridge, USA

\author{
EDITORIAL BOARD CHAIR \\ David Eisenbud \\ University of California \\ Berkeley, USA
}

BOARD OF EDITORS

Georgia Benkart

Dave Benson

Richard E. Borcherds

John H. Coates

J-L. Colliot-Thélène

Brian D. Conrad

Hélène Esnault

Hubert Flenner

Edward Frenkel

Andrew Granville

Joseph Gubeladze

Ehud Hrushovski

Craig Huneke

Mikhail Kapranov

Yujiro Kawamata

János Kollár

Yuri Manin

Barry Mazur

Susan Montgomery
University of Wisconsin, Madison, USA

University of Aberdeen, Scotland

University of California, Berkeley, USA

University of Cambridge, UK

CNRS, Université Paris-Sud, France

University of Michigan, USA

Universität Duisburg-Essen, Germany

Ruhr-Universität, Germany

University of California, Berkeley, USA

Université de Montréal, Canada

San Francisco State University, USA

Hebrew University, Israel

University of Kansas, USA

Yale University, USA

University of Tokyo, Japan

Princeton University, USA

Northwestern University, USA

Harvard University, USA

University of Southern California, USA
Shigefumi Mori

Andrei Okounkov

Raman Parimala

Victor Reiner

Karl Rubin

Peter Sarnak

Michael Singer

Ronald Solomon

Vasudevan Srinivas

J. Toby Stafford

Bernd Sturmfels

Richard Taylor

Ravi Vakil

Michel van den Bergh

Marie-France Vignéras

Kei-Ichi Watanabe

Andrei Zelevinsky

Efim Zelmanov
RIMS, Kyoto University, Japan

Princeton University, USA

Emory University, USA

University of Minnesota, USA

University of California, Irvine, USA

Princeton University, USA

North Carolina State University, USA

Ohio State University, USA

Tata Inst. of Fund. Research, India

University of Michigan, USA

University of California, Berkeley, USA

Harvard University, USA

Stanford University, USA

Hasselt University, Belgium

Université Paris VII, France

Nihon University, Japan

Northeastern University, USA

University of California, San Diego, USA

\section{PRODUCTION}

contact@msp.org

Silvio Levy, Scientific Editor

Andrew Levy, Production Editor

See inside back cover or www.jant.org for submission instructions.

The subscription price for 2011 is US \$150/year for the electronic version, and \$210/year (+ \$35 shipping outside the US) for print and electronic. Subscriptions, requests for back issues from the last three years and changes of subscribers address should be sent to Mathematical Sciences Publishers, Department of Mathematics, University of California, Berkeley, CA 94720-3840, USA.

Algebra \& Number Theory (ISSN 1937-0652) at Mathematical Sciences Publishers, Department of Mathematics, University of California, Berkeley, CA 94720-3840 is published continuously online. Periodical rate postage paid at Berkeley, CA 94704, and additional mailing offices.

ANT peer review and production are managed by EditFLOw ${ }^{\mathrm{TM}}$ from Mathematical Sciences Publishers.

PUBLISHED BY

mathematical sciences publishers

http://msp.org/

A NON-PROFIT CORPORATION

Typeset in LATEX

Copyright $\odot 2011$ by Mathematical Sciences Publishers 


\section{Algebra \& Number Theory}

\section{Volume $5 \quad$ No. $2 \quad 2011$}

On the Hom-form of Grothendieck's birational anabelian conjecture in positive characteristic

\section{MOHAMED SAÏDI and AKIO TAMAGAWA}

Local positivity, multiplier ideals, and syzygies of abelian varieties

RoBERT LAZARSFELD, GIUSEPPE PARESCHI and MiHNEA POPA

KATHERINE STANGE

The basic geometry of Witt vectors, I The affine case

JAMES BORGER

Correction to a proof in the article Patching and admissibility over two-dimensional complete local domains

DANNY NEFTIN and ELAD PARAN 